\title{
Social behavioral changes in MPTP-treated monkey model of Parkinson's disease
}

\author{
Elodie Durand ${ }^{1}$, Odile Petit ${ }^{2}$, Léon Tremblay ${ }^{3}$, Cédric Zimmer $^{2}$, Véronique Sgambato-Faure ${ }^{3}$, \\ Carine Chassain ${ }^{4}$, Marlène Laurent ${ }^{1}$, Bruno Pereira ${ }^{4}$, Céline Silberberg $^{1}$ and Franck Durif ${ }^{1}$ \\ 1 Université d'Auvergne Clermont 1, UFR Médecine, EA 7280 (NPsy-Sydo), Clermont-Ferrand, France \\ 2 Département Ecologie, Physiologie et Ethologie, Institut Pluridisciplinaire Hubert Curien, UMR 7178, CNRS-UDS, Strasbourg, France \\ ${ }^{3}$ Centre de Neurosciences Cognitives, UMR 5229 CNRS-Université Lyon 1, Bron, France \\ ${ }^{4} \mathrm{CHU}$ Clermont Ferrand, Gabriel Montpied, Clermont-Ferrand, France
}

\section{Edited by: \\ Angela Roberts, University of \\ Cambridge, UK \\ Reviewed by: \\ Michael Arthur Van Der Kooij, Ecole \\ Polytèchnique Féderale de \\ Lausanne, Switzerland \\ Julien Vezoli, Ernst Strüngmann \\ Institute for Neuroscience in \\ Cooperation with Max Planck \\ Society, Germany}

*Correspondence:

Elodie Durand, CHU Clermont

Ferrand/Service Neurologie Pr Durif,

58 rue Montalembert, 63003

Clermont-Ferrand, France

e-mail: edurand2@

chu-clermontferrand.fr
Parkinsonian patients experience not only the physical discomfort of motor disorders but also the considerable psychological distress caused by cognitive deficits and behavioral disorders. These two factors can result in a disruption of social relationships during the symptomatic and even the presymptomatic motor states of the disease. However, it remains difficult, if not impossible, to evaluate social relationships in presymptomatic patients. The present study focused on the evaluation of social relationships within a group of female long-tailed macaques during presymptomatic and symptomatic motor states induced by Chronic Low-Dose (CLD) and then Chronic High-Dose (CHD) systemic administration of 1-methyl-4-phenyl-I,2,3,6-tetrahydropyridine (MPTP). Dopaminergic denervation within basal ganglia and cortical areas was evaluated using Positron Emission Tomography (PET) scans with 18F-DOPA (6-[18F]-fluoro-L-3,4-dihydroxyphenylalanine) radiotracer. Interestingly, social behavioral changes could be identified in the presymptomatic motor state before any motor and/or cognitive impairment occurred. Stronger effects were observed in subordinate animals compared to dominant animals. From baseline state to CLD-presymptomatic motor state, the frequency of emitted affiliative and aggressive behaviors increased. From CLD-presymptomatic to CHD-presymptomatic motor states, the frequency of the three categories of social behaviors (aggressive, submissive and affiliative) decreased. At this time, quantitative data analysis in PET scans highlighted a dopaminergic denervation in the insula and the posterior caudate nucleus. Finally, the frequency of the three categories of social behaviors decreased during the stable-symptomatic motor state compared to baseline and presymptomatic motor states; this was also associated with motor and cognitive disorders and a dopaminergic denervation in all the evaluated cortical and subcortical structures.

Keywords: Parkinson's disease, social behavior, non-human primate model, MPTP, PET imaging

\section{INTRODUCTION}

Idiopathic Parkinson's disease (PD) is characterized by a loss of dopaminergic neurons in the substantia nigra pars compacta $(\mathrm{SNc})$, resulting in decreased levels about $60 \%$ of dopamine release in the striatum and hence causing motor symptoms (bradykinesia, tremor and rigidity) (Kish et al., 1988). However, it is clear that PD is not only a "motor" but also a "cognitive" and "neuropsychiatric" disease (Thobois et al., 2010; Gallagher and Schrag, 2012). Psychiatric disorders include apathetic state (Pedersen et al., 2009; Thobois et al., 2010), anxiety (Gallagher and Schrag, 2012) and depression (Martínez-Martín and Damián, 2010) as well as hypomania, psychosis and impulse control disorders observed in patients receiving dopaminergic treatment (Weintraub et al., 2006; Ulla et al., 2012). The pathophysiology of such disorders has not yet been completely understood but includes lesions of the dopaminergic, serotoninergic, and noradrenergic systems involved in Parkinson's disease (Hirsch et al.,
2003; Kish et al., 2008). However, a change in the mesocorticolimbic dopaminergic system could play a role in the behavioral disorders of PD (Remy et al., 2005). Indeed, in addition to the lesion of the dopaminergic nigrostriatal system, other dopaminergic systems are also damaged in PD, namely those originating in the ventral tegmental area (VTA) that project to the limbic system, which is involved in the reward circuit (mesolimbic system), and also to the prefrontal cortex, which is involved in personality traits (mesocortical system) (Tzschentke, 2001; Haber and Knutson, 2010).

The aforementioned non-motor symptoms could have an early impact on the social life of parkinsonian patients, leading some of them to become socially isolated both from themselves and from society. The symptoms impact the quality of life of parkinsonian patients, namely affecting social interactions, communication and/or emotion recognition (Schrag et al., 2000; Yoshimura et al., 2005; Pell et al., 2006). 
It is therefore legitimate to ask whether a lesion of the dopaminergic system could affect social interactions. What types of interactions could be affected by a dopaminergic lesion? Finally, which brain regions (basal ganglia and/or cortical areas) may be involved in social interactions changes? Answering these questions in patients is problematic, as it would be difficult if not impossible to accurately evaluate their social interactions on a daily basis and understand the real social impact of this disease. In this context, the use of a non-human primate model therefore appears useful to study the social impact of dopamine neuron loss in Parkinson's disease. In the wild, Macaca fascicularis females remain within their native group throughout their lives and therefore form clans of related individuals. Their ranks are more stable than those of males and are transmitted from mother to daughter forming a matrilineage that rarely changes over generations (Van Noordwijk and Van Schaik, 1987, 1999; Gumert, 2010). Any change in social behavior in a group of Macaca fascicularis might therefore impact the balance of inter-individual social relationships.

Several previous studies in non-human primates have shown the important role of several monoamines, such as dopamine, in hierarchical status and the expression of social behaviors in macaques (Redmond et al., 1971; Kaplan et al., 2002; Morgan et al., 2002; Riddick et al., 2009; Nader et al., 2012). Furthermore, neurobiological studies in non-human and human primates have also shown the role of cortico-limbic regions (namely the amygdala, the orbitofrontal cortex, the anterior cingular cortex and the insula) in the social behavioral network and in social cognition generally, which is heavily dependent upon the expression and recognition of emotions (Amaral, 2002; Machado and Bachevalier, 2006; Rushworth et al., 2007; Machado et al., 2009).

Currently, the primate model produced with the neurotoxin 1-methyl-4-phenyl-1,2,3,6-tetrahydropyridine (MPTP) is considered as the gold standard animal model of PD because of its close resemblance to PD. Indeed, this model, particularly with systemic MPTP administration in macaques, characteristically replicates marked cellular loss in the SNc, the cardinal motor symptoms of PD (including abnormalities in axial movements and postures), and the full extent of motor complications associated with chronic dopaminergic treatment (Jenner, 2003). Furthermore, the primate MPTP model can reproduce non-motor symptoms of PD including cognitive, sleep and gastrointestinal dysfunction (Barraud et al., 2009; Chaumette et al., 2009; Schneider et al., 2010). The chronic low-dose (CLD)-MPTP model of parkinsonism in non-human primates was specifically developed to study the presymptomatic motor state in early PD (Schneider and Kovelowski, 1990). This model induces a dopaminergic denervation in the SN/VTA (Schneider, 1990), dopaminergic frontostriatal cognitive deficits and deficits in spatial delayed response, set shifting, planning and impulsivity (Schneider and Kovelowski, 1990; Schneider and Roeltgen, 1993; Schneider and Pope-Coleman, 1995; Decamp and Schneider, 2004; Schneider, 2006; Vezoli et al., 2011) and recently sleep-wake disorders (Videnovic et al., 2014). Moreover, this CLD-MPTP model has been shown to induce a striatal denervation pattern more similar to the one observed in PD patients (Gibb and Lees, 1991; Perez-Otano et al., 1994) than the one induced by chronic high-dose or acute MPTP intoxication. Most previous studies using chronic high-dose administration of MPTP (CHD-MPTP), mainly focused on motor and cognitive disorders related to neurochemical, imaging or pharmacological findings (Chassain et al., 2001; Madras et al., 2006; Blesa et al., 2012; Neumane et al., 2012; Kortekaas et al., 2013). Interestingly, a longitudinal behavioral study in MPTP-hemiparkinsonian vervet monkeys also showed a link between changes in social behaviors (aggressive and affiliative) and striatal dopamine levels measured by ${ }^{18}$ F-DOPA (6-[18F]-fluoro-L-3,4-dihydroxyphenylalanine) PET (Positron Emission Tomography) scanning (Melega et al., 1996).

Therefore, the aim of the present study was to identify the impact of chronic exposure to low and then to high doses of MPTP on the social behavior of six female long-tailed macaques living in a social group, and to assess the time course evolution of cognitive and motor disabilities and social behavior disorder(s). PET (Positron Emission Tomography) scanning was used to assess dopaminergic denervation in basal ganglia and cortical areas using ${ }^{18} \mathrm{~F}-\mathrm{DOPA}$ (6-[18F]-fluoro-L-3,4-dihydroxyphenylalanine).

\section{MATERIALS AND METHODS ETHICS STATEMENT}

All procedures were carried out according to National Institute of Health and the European Directive 2010/63/EU guidelines and the Department of Veterinary Services (DDSV Clermont Ferrand, France). These experiments were also carried out according to guidelines published in the Guide for the Care and Use of Laboratory Animals of the National Institutes of Health. Specific authorization covering this study was delivered by the regional animal ethical committee (Comité d'Ethique en Expérimentation Animale Auvergne, C2EA-02) under Permit Number: CE19-08.

\section{ANIMALS}

Experiments were conducted on six female long-tailed macaques (Macaca fascicularis) (5.2-6.6 years old, weighing $3.6-5.6 \mathrm{~kg}$ at the beginning of the study): monkeys A, B, C, D, E, and F. This number of animals was sufficient for statistical analysis of social behaviors. They were housed together throughout the study, had free access to water and received food twice a day. No animals were sacrificed during the study. Standard conditions of humidity $(55 \pm 10 \%)$, temperature $\left(24 \pm 2^{\circ} \mathrm{C}\right)$ and light (12-h light/dark cycles) were respected. The housing consisted of two rooms connected by a trap door that was left open at all times, each room consisted of three $1.8 \mathrm{~m}^{3}$ cages $(1 \times 1 \times$ $1.8 \mathrm{~m}$ ). Each animal therefore had access to a minimum of $1.8 \mathrm{~m}^{3}$, as required by National Institute of Health and the European Directive 2010/63/EU guidelines.

\section{CONTRACEPTIVE IMPLANT}

Several studies have already demonstrated an effect of menstrual cycle on social behavior (Adams et al., 1985; Michael and Zumpe, 1993; Czoty et al., 2009). In order to remove any such influence, all animals $(n=6)$ were fitted with the subdermal contraceptive implant (half an implant/animal), Implanon ${ }^{\circledR}$ (Schering-Plough, USA) primarily used in humans (Isley, 2010) prior to all testing sessions and the evaluation of baseline conditions. This implant 
overcomes the effects of menstrual cycles on observed behaviors and monoamines. Each Implanon ${ }^{\circledR}$ rod consists of an ethylene vinylacetate copolymer core, containing $68 \mathrm{mg}$ of the synthetic progestin etonogestrel and produces an effect for 3 years.

\section{TOXIN ADMINISTRATION}

All animals $(n=6)$ were exposed to MPTP hydrochloride (MPTP-HCl) (dissolved in saline, Sigma, St. Louis, USA) by systemic intramuscular (i.m.) administration. Firstly, animals received $0.1 \mathrm{mg} / \mathrm{kg}$ every $4-5$ days for 58 weeks $(10.7-12.3 \mathrm{mg} / \mathrm{kg}$ ), i.e., chronic low dose CLD-MPTP protocol, to study the CLDpresymptomatic motor state. A second protocol was then used (6 days after the final CLD-MPTP injection), in which MPTP was injected once per week at $0.4 \mathrm{mg} / \mathrm{kg}$ under light anesthesia using ketamine $(0.5 \mathrm{mg} / \mathrm{kg}$ ) (Neumane et al., 2012), i.e., chronic high-dose CHD-MPTP protocol, to study the stable-symptomatic motor state, although a short CHD-presymptomatic motor state had still been studied. During this second protocol, animals were administered 3-17 doses for a period of 4-24 weeks (1.0$6.5 \mathrm{mg} / \mathrm{kg}$ ) (Table 1; Figure 1).

\section{EXPERIMENTAL DESIGN}

Animals had been previously trained for cognitive testing, and their social and motor behaviors were assessed over the 5 months preceding MPTP administration in order to have a global representation of the baseline state. One testing session was carried out at least $36 \mathrm{~h}$ after MPTP injection at 2-3 week intervals during the CLD-presymptomatic period and weekly during the CHD-presymptomatic and stable-symptomatic periods. Thus, 20 testing sessions were performed during the CLDpresymptomatic motor state. During the CHD-presymptomatic motor state, according to the animals, 2-10 testing sessions were performed and during the stable-symptomatic motor state 3 testing sessions were assessed. These social, motor and cognitive evaluations were carried out over 4-day testing sessions (Day $1=1 \mathrm{~h}$ motor behavior assessment ( 2 animals $)+$ cognitive testing, Day $2=1 \mathrm{~h}$ motor behavior assessment ( 2 animals $)+6 \mathrm{~h}$ social behavior assessment, Day $3=1 \mathrm{~h}$ motor behavior assessment $(2$ animals $)+$ cognitive testing, Day $4=6 \mathrm{~h}$ social behavior assessment) (Figure 1).

\section{BEHAVIORAL STUDY}

No experimenters were present in the testing room [three $1.8 \mathrm{~m}^{3}$ cages $(1 \times 1 \times 1.8 \mathrm{~m})$ with separating grids removed to obtain an aviary system] during social and motor assessments. This was achieved through the use of three black and white wall cameras connected to a server and equipped with a Smart Digital Video Recorder for Life Sciences, also known as "Numeriscope" (View Point, Lyon, France). In addition, a color camera fixed to the ceiling and rotating $360^{\circ}$ (View Point, Lyon, France) tracked animal movements in real-time and specifically zoomed in on the faces of the animals to discriminate different facial expressions.

Table 1 | Individual sensitivity to MPTP during the CLD-MPTP and the CHD-MPTP protocols (number of MPTP injections, total amount of MPTP administered $(\mathrm{mg} / \mathrm{kg}$ ) and related clinical score).

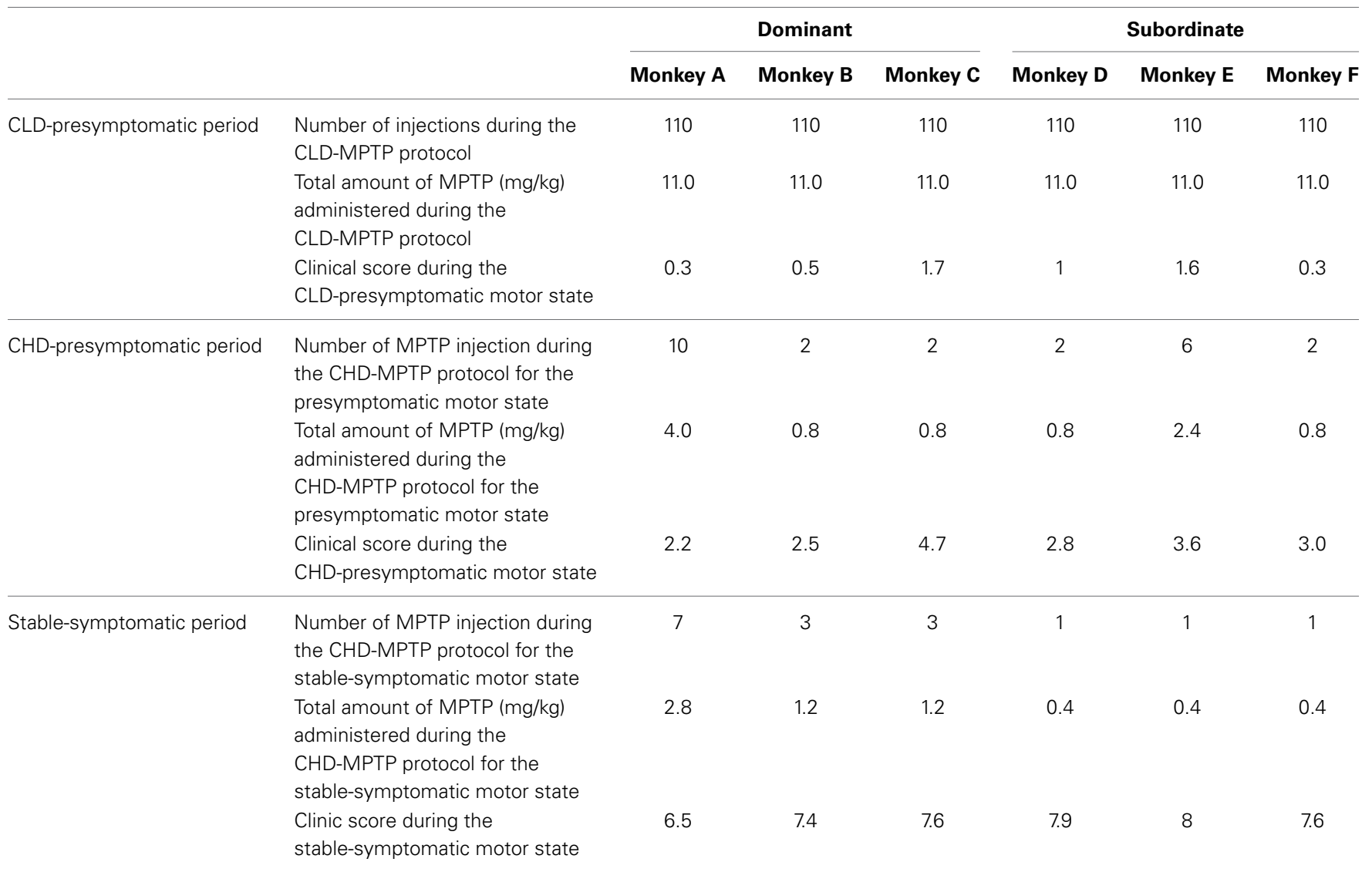




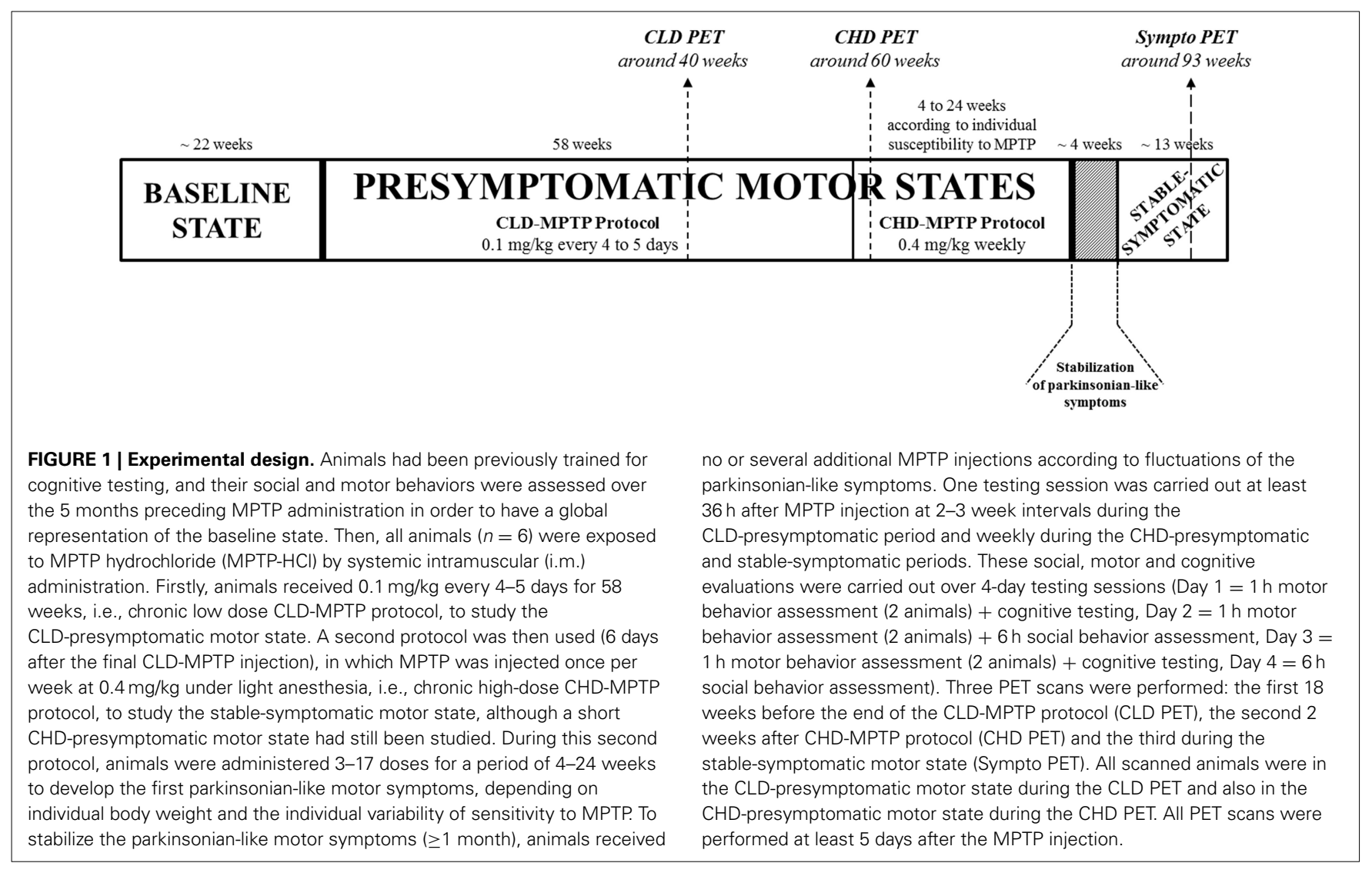

Each evaluation session was recorded to allow evaluations by two experimenters with no previous knowledge of the state of the animal.

\section{Motor assessment}

Video observation was used to evaluate animals, and motor behavior was scored five times during the 1-h protocol in order to have an average score for each motor test. Two animals, housed separately in adjacent cages $(1 \times 1 \times 1.8 \mathrm{~m}$ for each cage $)$, could be evaluated simultaneously and independently.

Clinical rating scale. Clinical rating was inspired from the Canadian rating scale (Gomez-Mancilla et al., 1993; Chassain et al., 2001). Clinical symptoms data presented the total score for posture $(0=$ normal; $1=$ intermittent flexion of trunk and limbs; $2=$ constant flexion of trunk and limbs; $3=$ crouch position), mobility ( $0=$ normal; $1=$ mild decrease; $2=$ moderate decrease; $3=$ severe decrease $)$, gait $(0=$ normal; $1=$ slow; $2=$ very slow; 3 = very slow with freezing $)$ and tremor $(0=$ absent; $1=$ mild postural tremor; $2=$ moderate postural tremor; $3=$ resting tremor) (maximum total score of 12). A clinical score $>0$ and $=6$, was defined as the presymptomatic motor state; finally for a clinical score $>6$, the individual was considered to be developing parkinsonian-like motor symptoms in stable-symptomatic motor state (Chassain et al., 2001).

Locomotor activity assessment. Locomotor activity was assessed using a Vigie Primates ${ }^{\circledR}$ image analyzer system (View Point, Lyon,
France) (Chassain et al., 2001). The system was comprised of a video camera connected to a video image analyzer system that calculated the quantity and quality of the locomotor activity in real time. The images were digitized with a $800 \times 600$ pixel definition on 256 gray levels, and the changes in gray level in pixels from one image to the next were counted every $80 \mathrm{~ms}$ to plot a raw activity curve (Chassain et al., 2001). It was possible to change the following parameters: (i) the detection sensitivity determining the threshold from which a pixel is considered to have changed from one image to the next, (ii) the duration of data acquisition before obtaining a summary of the activities of the animal in question during that period, and (iii) the duration of the experiment, which could range from one second to several days (a 1-h testing session during this study). From the raw curve, the activity of each animal could be separated into three states. The first state corresponded to inactivity of the animal, the second to normal activity and the third to hyperactivity. Thresholds between the three states could be adjusted to discriminate between the movements of the animal. During this study the time spent in an inactive state was specifically evaluated to ensure a good correlation between increased periods of inactivity and clinical motor disorders (Chassain et al., 2001).

\section{Social assessment}

Dominance hierarchy. Hierarchical stability was verified over the 5 months preceding the first MPTP injection. It was measured via the tabulation of unidirectional conflicts and avoidances into a matrix of agonistic behaviors (aggressive and submissive 
behaviors) which were then reorganized into a dominance matrix (Angst, 1975; Bentley-Condit and Smith, 1999; Riddick et al., 2009).

Hierarchical status was assessed by the hierarchical steepness value, which shows the size of the absolute differences in overall dominance success between individuals of adjacent ranks. In other words, the steeper the slope between two individuals, the stronger the difference between two dominance ranks will be. The slope was measured using SOCPROG.6 software (Whitehead, 2009). Individual hierarchical status ranking was determined using the David's score for each animal (Gammell et al., 2003). The most dominant animal (monkey A) presented the highest David's score and the most subordinate animal (monkey F) presented the lowest value. The dominance hierarchy of the social group was linear and organized as followed: monkey $\mathrm{A}>$ monkey $\mathrm{B}>$ monkey $\mathrm{C}>$ monkey $\mathrm{D}>$ monkey $\mathrm{E}>$ monkey F.

Social observations. In this study, 19 social behaviors were assessed and were categorized as aggressive, submissive or affiliative behaviors (Van Hooff, 1967; Morgan et al., 2000; Brent and Veira, 2002; Kaplan et al., 2002; Camus et al., 2013) (Figure 2). All behaviors required animal movement (displacement, stiff approach, lunge, chase, avoid) and some required facial expressions (open mouth display, stare, silent bared teeth display, teeth chatter, lipsmack display). For each animal, all social behaviors were recorded for $2 \mathrm{~h}$ using the focal animal sampling method (Altmann, 1974) and the LabWatcher software (View Point, Lyon, France). In these focal group sessions, each behavior was recorded in terms of emission and reception. The average frequency of the three categories of behavior over a period of $2 \mathrm{~h}$ was shown for each of the three motor states (normal, CLD-presymptomatic following CLD-MPTP protocol, CHD-presymptomatic following CHD-MPTP protocol and stable-symptomatic). The assessment order of each individual social behavior was randomly defined using the Kendall and BB Smith Table. All observations were carried out between 8.00 a.m. and 2.00 p.m. Animals were fed with fruit and vegetables at $7.30 \mathrm{am}$, and had ad libitum access to pellets and water during social behavior evaluation.

\begin{tabular}{|c|l|c|}
\hline Category & \multicolumn{2}{|c|}{ Behavior } \\
\hline Aggression & $\begin{array}{l}\text { bite } \\
\text { chase } \\
\text { displacement } \\
\text { grab } \\
\text { lunge } \\
\text { open mouth display } \\
\text { slap } \\
\text { stare } \\
\text { stiff approach } \\
\text { Submission }\end{array}$ & $\begin{array}{l}\text { avoid } \\
\text { shrink } \\
\text { silent bared teeth display } \\
\text { teeth chatter } \\
\text { Affiliation }\end{array}$ \\
\hline $\begin{array}{l}\text { Open mouth display \& Stare } \\
\text { clasp } \\
\text { groom the head } \\
\text { lipsmack display } \\
\text { mouth approach } \\
\text { presentation }\end{array}$ \\
\hline
\end{tabular}

FIGURE 2 | Social behaviors of long-tailed macaques. Social behaviors of long-tailed macaques are split into three categories: Aggression, Submission and Affiliation. In this study, 19 social behaviors were assessed, some of which required motor abilities (i.e., all facial expressions: open mouth display, stare, silent bared teeth display, teeth chatter, lipsmack display), and all of which required animal movement (displacement, stiff approach, lunge, chase, avoid). 


\section{COGNITIVE STUDY: ORDT (OBJECT RETRIEVAL DETOUR TASK)}

The ORDT was used to assess the ability of the animals to retrieve an object (a piece of fruit) from inside a transparent box that only opened on one side (Taylor et al., 1990; Roeltgen and Schneider, 1991; Schneider and Pope-Coleman, 1995; Palfi et al., 1996; Vezoli et al., 2011). The Plexiglas box $(15 \times 15 \times 5 \mathrm{~cm})$ was fixed on a tray that was adapted to the home cage of the animal. The experimenter modified the cognitive level and motor skills required to solve the task and retrieve the piece of fruit by varying the location of the box in relation to the subject, the location of the reward inside the box, and finally the orientation of the open side of the box in relation to the subject. As shown in Supplementary Material, each testing session consisted of 35 trials that were randomly presented to the animals. This testing session contained "difficult" or "detour" trials in which the animal had to make a detour around a closed side of the box to reach the reward (detour to reach the reward: see configurations 1-20 in Supplementary Material), and "easy" trials which were defined as trials in which the opening of the box was facing the animal (no detour to reach the reward: see configurations 21-35 in Supplementary Material). Subjects were allowed $1 \mathrm{~min}$ to retrieve the reward, and then the box was set up for the next trial. The movements and responses of the animal involving the tray or the box were not restrained in any way.

Measures of performance included the number of successes and errors on detour or easy trials. Successes (retrieval of reward on the first reach) were expressed as a percentage of the total number of detour and easy trials. Errors (barrier hits, i.e., hitting a transparent side of the box) were expressed as percentage of responses observed (there could be several responses per trial except in the case of success) for easy or detour trials.

Four of the six animals were trained to come and work in the testing cage, without being forced in any way. Animals were trained during 11-18 sessions to achieve the cognitive task with a minimum of $75 \%$ successfully trials during three consecutive sessions. The other 2 animals (the third dominant animal: monkey $\mathrm{C}$ and the second subordinate animal: monkey E) refused to work on this test despite several weeks of training.

\section{IMAGING STUDY: PET SCANS PET scan design}

Owing to limited access to the PET facility, only 4 animals of the social group were selected. On the one hand, the 3 animals with the greatest behavioral social changes, namely the three most subordinate animals, were selected for the imaging part of the study, waiting for variation of ${ }^{18} \mathrm{~F}$-DOPA uptake (Ki values) in the brain structures explored. On the other hand, the most dominant animal was also selected because of its essential role in the stability of the social group (Petit and Thierry, 1992). Three PET scans were performed: the first 18 weeks before the end of the CLD-MPTP protocol (CLD PET), the second 2 weeks after CHD-MPTP protocol (CHD PET) and the third during the stable-symptomatic motor state (Sympto PET). All scanned animals were in the CLDpresymptomatic motor state during the CLD PET and also in the CHD-presymptomatic motor state during the CHD PET. All PET scans were performed at least 5 days after the MPTP injection. Since no PET scan could be performed during the baseline state, all data obtained from the three PET scans for the four MPTPtreated animals were compared to data from PET scan performed in five randomly selected healthy animals (Control PET), considered as basal levels data. Finally, we compared the three PET scans performed for the four MPTP-treated animals.

It was important to note here that the measurement of ${ }^{18} \mathrm{~F}$ DOPA uptake in the control healthy animas showed less than $10 \%$ variability, except in the subtantia nigra (13\%), although no information was available regarding their hierarchical status. Moreover, previous PET studies with test-retest data have shown a good reproducibility of radiotracer binding measurement (Costes et al., 2007; Ballanger et al., 2013).

\section{Acquisitions}

Animals were fasted overnight prior to MRI (Magnetic Resonance Imaging) and PET exams. On the day of the experiment, animals were pretreated with Atropine $(0.05 \mathrm{mg} / \mathrm{kg}$ i.m. $)$ and were anesthetized with Zoletil (15 mg/kg i.m.) 15 min later. Lactated Ringer's solution was continuously infused through a saphenous vein catheter. Animals were then transported to the Imaging Center (CERMEP, Lyon, France) where they were placed on a stereotaxic apparatus. Respiratory frequency, $\mathrm{pO} 2$ and heart rate were monitored throughout the experiment. One hour after the anesthesia, PET scans were performed in a three-dimensional (3D) mode using a Siemens CTI HR+ tomograph, with an axial field of view of $15.2 \mathrm{~cm}$, yielding 63 planes and a nominal inplane resolution of $4.1 \mathrm{~mm}$ full width at half maximum (FWHM). Before the tracer injection, a transmission scan $68 \mathrm{Ge}$ rotating rod sources; $10 \mathrm{~min}$ ) was acquired to correct for tissular $511 \mathrm{keV}$ gamma attenuation. Dynamic acquisition started with the intravenous (i.v.) injection of ${ }^{18} \mathrm{~F}$-DOPA (138 $\left.\pm 7.4 \mathrm{MBq}\right)$ (Neumane et al., 2012).

\section{PET data processing and analysis}

The 3D emission data were reconstructed with attenuation and scatter correction by a 3D filtered backprojection algorithm (Hamming filter; cut-off frequency, 0.5cycles/pixel) and a zoom factor of three, giving a transaxial resolution of $6.5 \mathrm{~mm}$ FWHM. Reconstructed volumes were $128 \times 128$ matrices of $0.32 \times 0.32 \mathrm{~mm}^{2}$ pixels in sixty-three $2.42 \mathrm{~mm}$ spaced planes. The extraction of tissue time-activity concentration curves from automated delineated regions of interest was made possible by the specific Macaca fascicularis brain atlas (Ballanger et al., 2013). The influx rate constant of ${ }^{18} \mathrm{~F}-\mathrm{DOPA}(\mathrm{Ki})$ was calculated by linearization of the graphical Patlak plot (Patlak et al., 1983) over $90 \mathrm{~min}$ post-injection using the cerebellum as the reference region.

During this study, a limited number of areas with significant ${ }^{18}$ F-DOPA uptake (Ki values) were assessed. Areas within the Basal Ganglia were the Anterior and Posterior Caudate Nucleus (Ant CdN/Post CdN), the Anterior Putamen (Ant Put), the ventral striatum (V Str), the Posterior Dorsal and Ventral Putamen (Post D Put/Post V Put) and the Substantia Nigra $(\mathrm{SN})$. Areas assessed at the cortical level were the Insula (Ins), the Orbitofrontal Cortex (OFC), the Anterior Cingulate Cortex (ACC) and the Amygdala (Amyg). The cerebellum was the brain region reference. 


\section{STATISTICAL ANALYSIS}

Statistical analyses were performed with Statistica statistical software (version 10; StatSoft, Inc. Tulsa, USA). After verifying that the data followed a normal distribution, we carried out Analysis of Variance with Repeated Measures followed by the Newman-Keuls post-hoc test for social, cognitive and locomotor activity data. Clinical motor score data were analyzed using a paired Student $t$-test. PET scans data analysis between groups were performed with Random Effects Models, making it possible to consider both the group as fixed effect and the intra-subject variability with subject as random effects. PET scans intra-group data analysis in the MPTP-treated animals were performed with $\mathrm{Chi}^{2}$-test. The correlations between measures were calculated using Pearson's correlation. In all cases, significance was accepted at the $95 \%$ of confidence level $(p<0.05)$.

\section{RESULTS}

\section{MOTOR BEHAVIOR ANALYSES}

No animals developed any parkinsonian-like motor symptoms during the CLD-MPTP protocol. The clinical score was below the threshold score of 6 . No changes were observed in the time of inactivity from the baseline to the CLD-presymptomatic motor states (Figures 3A,B).

During the CHD-MPTP protocol following the CLD-MPTP protocol, two periods were observed. The first period corresponded to the CHD-presymptomatic motor state, and included a significant increase in the clinical score compared to the CLDpresymptomatic motor state $(3.1 \pm 0.9$ vs. $0.9 \pm 0.6 ; t=11.28$, $p<0.001$ ), although the score stayed below the threshold value of 6. No significant increase in the time of inactivity was observed during this state. The second period corresponded to the appearance of stable parkinsonian-like motor symptoms, and a significant increase of clinical score was observed in comparison to the CHD-presymptomatic (7.5 \pm 0.6 vs. $3.1 \pm 0.9 ; t=13.26$, $p<0.001)$ and the CLD-presymptomatic $(0.9 \pm 0.6 ; t=29.97$, $p<0.001)$ motor states. At this state, all animals had a clinical score $>6$. The time spent in inactivity during this period was positively correlated with clinical score $(r=0.69, p<0.001)$ and was significantly higher than the CHD-presymptomatic [3510.9 \pm $81.1 \mathrm{~s}$ vs. $2853.6 \pm 435.0 \mathrm{~s} ; F_{(3,20)}=9.69, p<0.001$; post-hoc test, $p<0.01$ ] and the CLD-presymptomatic [2633.2 $\pm 644.5 \mathrm{~s}$; $F_{(3,20)}=9.69, p<0.001$; post-hoc test, $\left.p<0.01\right]$ motor states (Figures 3A,B). No significant difference was observed between the two subgroups (Dominant vs. Subordinate). However, high individual variability was observed in the sensitivity to MPTP, with varying numbers of CHD-MPTP injections and amounts of MPTP required to develop parkinsonian-like motor symptoms. Indeed, monkeys B, C, D, and F seemed to be more sensitive to MPTP than monkeys A and E (Table 1).

\section{SOCIAL ANALYSES}

\section{Baseline state}

The baseline frequency of agonistic behaviors, i.e., aggressive behaviors (emitted: $24.2 \pm 23.3$; received: $14.2 \pm 11.3$ ) and submissive behaviors (emitted: $34.1 \pm 27.2$; received: $32.3 \pm 37.8$ ), reflected the formal dominance within the social group, i.e., each animal recognized its own social status and that of its congeners

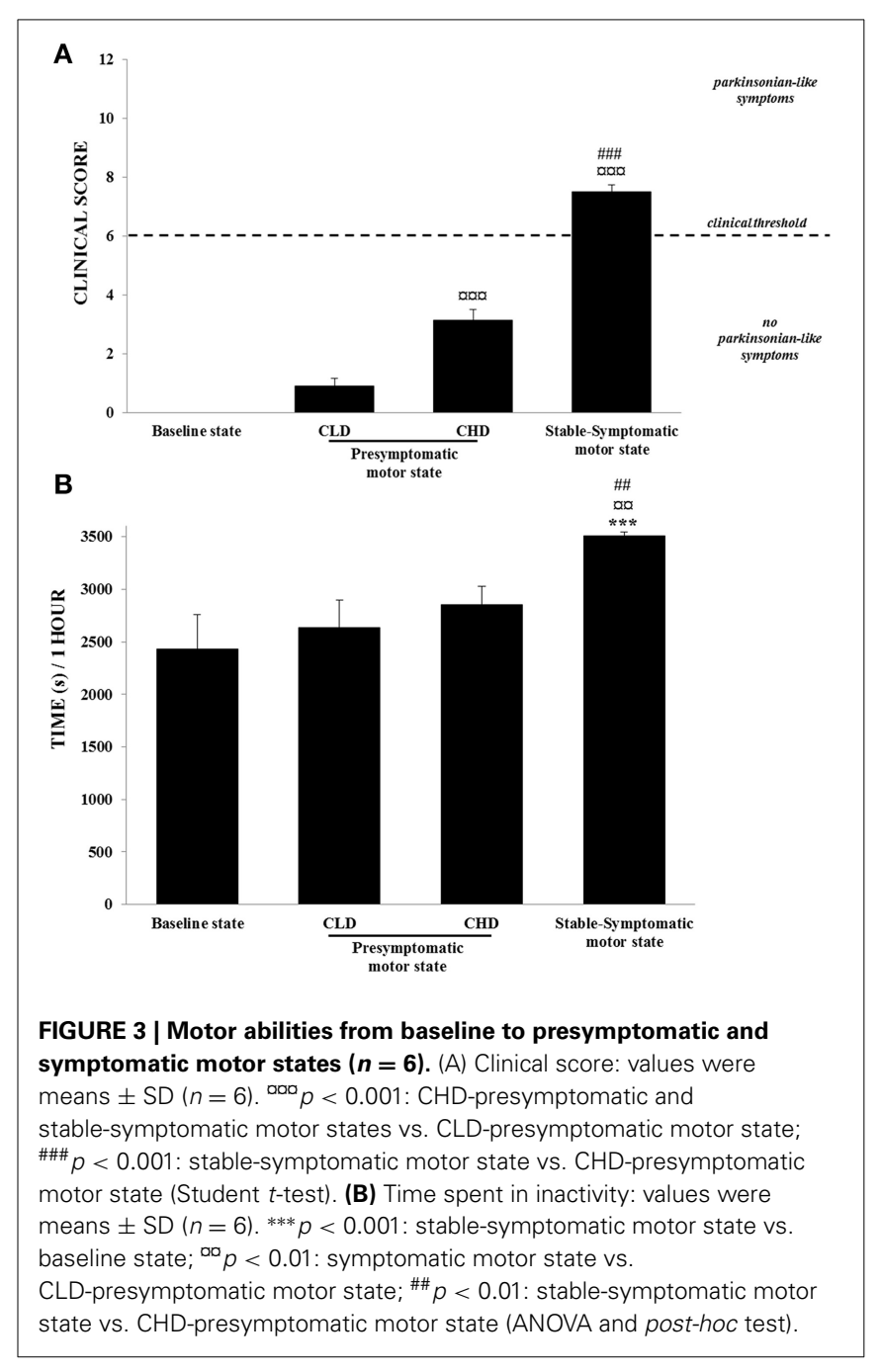

(Figures 4A,B). The affiliative behaviors category showed the highest frequency of appearance (emitted: $59.7 \pm 26.7$; received: $54.3 \pm 26.2$ ) (Figure 4C).

\section{CLD-presymptomatic motor state}

There was no significant change in the frequency of emitted and received aggressive behaviors from the CLD-presymptomatic motor state compared to the baseline state, although a marked increase was observed (emitted: $53.5 \pm 32.2$ vs. $24.2 \pm 23.3$; received: $35.7 \pm 46.2$ vs. $14.2 \pm 11.3$ ) (Figure $4 \mathrm{~A}$ ). No significant changes were seen in the frequency of emitted and received submissive behaviors (Figure 4B). The frequency of emitted affiliative behaviors significantly increased during the CLD-presymptomatic motor state compared to the baseline state $\left[83.1 \pm 27.7\right.$ vs. $59.7 \pm 26.7 ; F_{(3,20)}=4.02, p<0.05$; post-hoc test, $p<0.05$ ] (Figure 4C).

In subgroup social analyses, there was no significant change in the frequency of emitted and received aggressive behaviors. However, a marked increase in the frequency of emitted and received aggressive behaviors in subordinate animals was observed during the CLD-presymptomatic motor state compared 


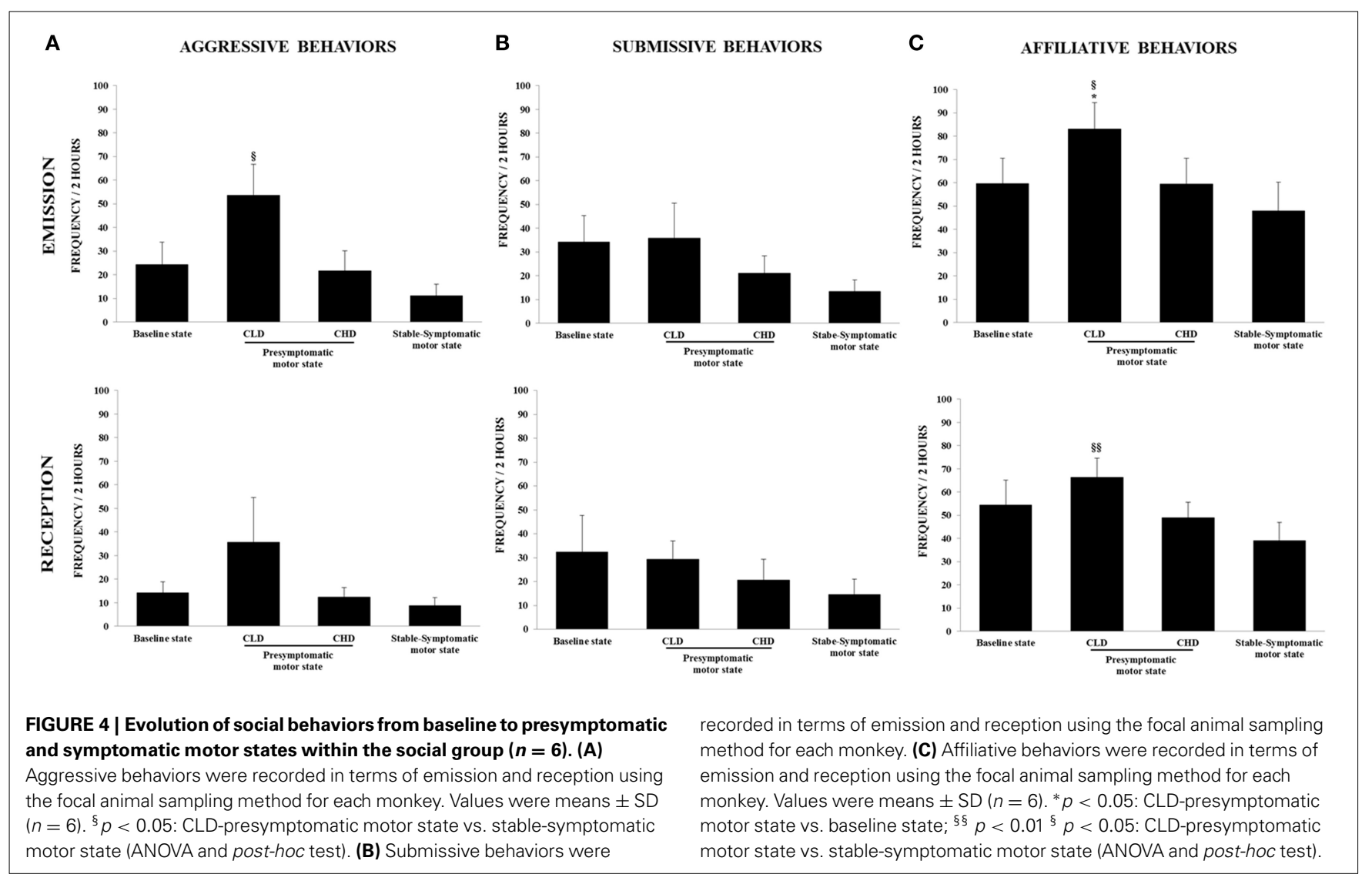

to the baseline state (emitted: $60.2 \pm 47.6$ vs. $9.8 \pm 11.6$; received: $62.6 \pm 55.0$ vs. $24.0 \pm 3.1$ ) (Figure $5 \mathrm{~A}$ ). No significant changes were seen in the frequency of emitted and received submissive behaviors whatever the social subgroup (Figure 5B). No change occurred in the frequency of emitted affiliative behaviors of dominant animals. Unlike dominant animals, the frequency of emitted affiliative behaviors of subordinate animals significantly increased during the CLD-presymptomatic motor state compared to the baseline state $\left[102.6 \pm 13.4\right.$ vs. $63.8 \pm 41.1 ; F_{(3,8)}=9.43, p<$ 0.05 ; post-hoc test, $p<0.05]$. No significant change occurred in the frequency of received affiliative behaviors of dominant and subordinate animals (Figure 5C).

Individual data (Table 2) were used to determine the interindividual involvement of each animal in these marked changes observed during the CLD-presymptomatic motor state compared to the baseline state. Monkey A, the most dominant animal, presented no major variation in its frequency of emitted aggressive behaviors in terms of emission or reception. Moreover, whilst its frequency of emitted affiliative behaviors increased almost twofold, no variation was noted in its frequency of received affiliative behaviors. Monkey B, the second most dominant animal, presented no major variation in its frequency of emitted aggressive behaviors but the reception of aggressive behaviors was half as high as the baseline values. Moreover, it did not present any major variation in its frequency of affiliative behaviors in terms of emission or reception. The four others animals in the social group showed a higher variation in the frequency of aggressive behaviors. Indeed, the frequency of emitted aggressive behaviors in monkey C increased almost three-fold, whilst its frequency of received aggressive behaviors increased six-fold. Moreover, it did not show any major variation of its frequency of affiliative behaviors, in terms of emission and reception. For monkey D, its frequency of emitted aggressive behaviors decreased about 3.5 times whilst the frequency of received aggressive behaviors increased almost five-fold. Moreover its frequency of emitted affiliative behaviors increased almost two-fold and no major variation was observed in its frequency of received affiliative behaviors. Monkey E presented a marked increase of about 14.5 times its frequency of emitted aggressive behaviors and did not show any variation in its frequency of received aggressive behaviors. Moreover, it did not shown any major variation in its frequency of emitted affiliative behaviors but its frequency of received affiliative behaviors increased almost two-fold. Finally, the most subordinate animal, monkey F, surprisingly emitted aggressive behaviors that had not been observed during the baseline state, and did not show any major variation in its frequency of received aggressive behaviors. Moreover, it showed a three-fold increase in its frequency of emitted affiliative behaviors and its frequency of received affiliative behaviors increased almost two-fold.

Interestingly, the dominance hierarchy within the social group was modified during the CLD-presymptomatic motor state $(A>B>C>E>F>D)$ compared to the baseline state $(\mathrm{A}>\mathrm{B}>\mathrm{C}>\mathrm{D}>\mathrm{E}>\mathrm{F})$. Thus, monkey $\mathrm{D}$ presented the lowest hierarchical status. 


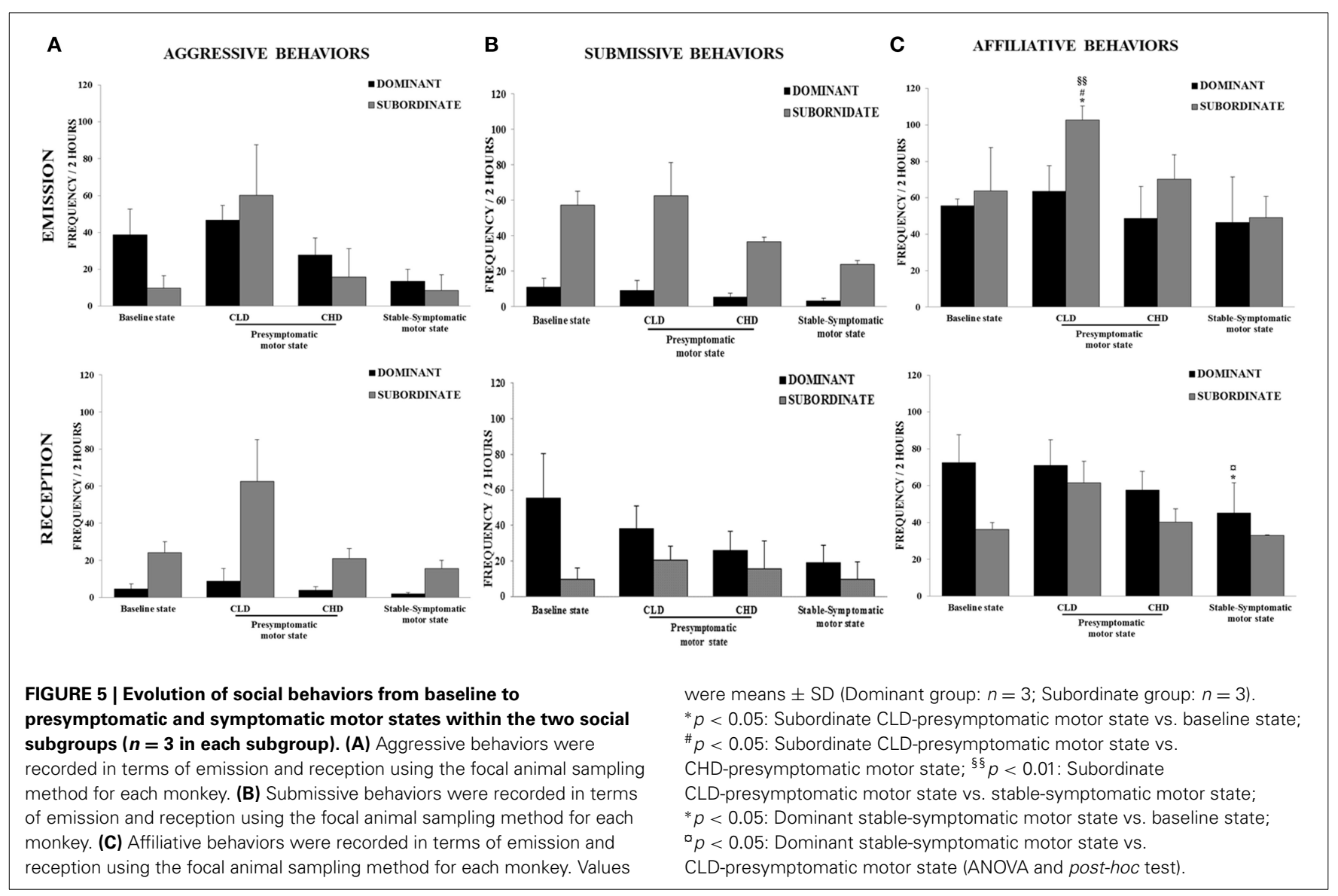

\section{CHD-presymptomatic motor state}

There was no significant change in the frequency of emitted and received aggressive behaviors compared to the baseline and the CLD-presymptomatic motor states, although a marked decrease was observed during the CHD-presymptomatic motor state compared to the CLD-presymptomatic motor state (emitted: $21.7 \pm 20.7$ vs. $53.5 \pm 32.2$; received: $12.4 \pm 10.0$ vs. $35.7 \pm$ 46.2) (Figure 4A). No significant changes were seen in the frequency of emitted and received submissive behaviors. However, a decrease in the frequency of emitted submissive was observed during the CHD-presymptomatic motor state compared to the CLD-presymptomatic motor state $(21.1 \pm 17.5$ vs. $35.8 \pm 36.3)$ (Figure 4B). There was no significant change in the frequency of emitted and received affiliative behaviors compared to the baseline and the CLD-presymptomatic motor states, although a decrease was observed during the $\mathrm{CHD}$-presymptomatic motor state compared to the CLD-presymptomatic motor state (emitted: $59.4 \pm 27.1$ vs. $83.1 \pm 27.7$; received: $48.9 \pm 16.6$ vs. $66.3 \pm$ 20.6) (Figure 4C).

In subgroup social analyses, there was no significant change in the frequency of emitted and received aggressive behaviors. However, a marked decrease in the frequency of emitted and received aggressive behaviors in subordinate animals was observed during the CHD-presymptomatic motor state compared to CLD-presymptomatic motor state (emitted: $15.7 \pm$ 26.7 vs. $60.2 \pm 47.6$; received: $21.0 \pm 3.8$ vs. $62.6 \pm 55.0$ ). No significant change in the frequency or emitted and receive submissive behaviors was observed. However, a marked decrease in the frequency of emitted submissive behaviors in subordinate animals was observed during the CHD-presymptomatic motor state compared to the CLD-presymptomatic motor state $(36.7 \pm 4.1 \mathrm{vs.}$ $62.6 \pm 32.3)$. No significant change in the frequency or emitted and receive affiliative behaviors was observed. However, a marked decrease in the frequency of emitted affiliative behaviors in subordinate animals was observed during the CHD-presymptomatic motor state compared to the CLD-presymptomatic motor state $(70.2 \pm 23.4$ vs. $102.6 \pm 13.4)$.

Individual data (Table 2) were used to determine the interindividual involvement of each animal in these marked changes observed during the CHD-presymptomatic motor state compared to the CLD-presymptomatic motor state. Monkey A, the most dominant animal, presented a decrease about 3 times in its frequency of emitted aggressive behaviors and did not show any variation in its frequency of received aggressive behaviors. No variation in its frequency of emitted submissive behaviors was observed, whilst its frequency of received submissive behaviors decreased almost two-fold. Finally, monkey A did not present any variation in its frequency of affiliative behaviors in terms of emission and reception. Monkey B, the second most dominant animal, presented no major variation in its frequency of aggressive and submissive behaviors in terms of emission and reception. Moreover, its frequency of emitted affiliative behaviors 
Table 2 | Individual frequency of the three categories of social behaviors during the baseline state, the CLD and CHD-presymptomatic motor states and the stable-symptomatic motor state.

\begin{tabular}{|c|c|c|c|c|c|c|c|}
\hline & & \multicolumn{3}{|c|}{ Dominant } & \multicolumn{3}{|c|}{ Subordinate } \\
\hline & & Monkey A & Monkey B & Monkey C & Monkey D & Monkey E & Monkey F \\
\hline \multirow{4}{*}{$\begin{array}{l}\text { Frequency of EMITTED } \\
\text { AGGRESSIVE BEHAVIORS }\end{array}$} & Baseline state & 58.0 & 46.7 & 11.3 & 22.7 & 6.7 & 0.0 \\
\hline & CLD-presymptomatic motor state & 47.8 & 60.0 & 32.5 & 6.5 & 97.0 & 77.2 \\
\hline & CHD-presymptomatic motor state & 17.1 & 46.0 & 19.7 & 46.5 & 0.6 & 0.0 \\
\hline & Stable-symptomatic motor state & 26.3 & 6.0 & 8.3 & 25.3 & 0.3 & 0.0 \\
\hline \multirow{4}{*}{$\begin{array}{l}\text { Frequency of RECEIVED } \\
\text { AGGRESSIVE BEHAVIORS }\end{array}$} & Baseline state & 0.0 & 9.3 & 4.0 & 24.7 & 26.7 & 20.7 \\
\hline & CLD-presymptomatic motor state & 0.1 & 4.2 & 22.0 & 126.1 & 28.5 & 33.3 \\
\hline & CHD-presymptomatic motor state & 0.1 & 7.0 & 4.3 & 21.5 & 17.0 & 24.5 \\
\hline & Stable-symptomatic motor state & 0.0 & 2.7 & 2.7 & 15 & 11.3 & 20.7 \\
\hline \multirow{4}{*}{$\begin{array}{l}\text { Frequency of EMITTED } \\
\text { SUBMISSIVE BEHAVIORS }\end{array}$} & Baseline state & 1.3 & 17.3 & 14.7 & 44.7 & 72.0 & 54.7 \\
\hline & CLD-presymptomatic motor state & 0.6 & 6.7 & 19.7 & 99.3 & 39.1 & 49.3 \\
\hline & CHD-presymptomatic motor state & 1.1 & 8.0 & 7.3 & 38.0 & 32.1 & 40.0 \\
\hline & Stable-symptomatic motor state & 0.0 & 4.7 & 4.7 & 20.7 & 22.0 & 28.3 \\
\hline \multirow{4}{*}{$\begin{array}{l}\text { Frequency of RECEIVED } \\
\text { SUBMISSIVE BEHAVIORS }\end{array}$} & Baseline state & 96.7 & 58.7 & 10.0 & 22.7 & 5.3 & 0.7 \\
\hline & CLD-presymptomatic motor state & 52.8 & 48.6 & 12.4 & 8.9 & 35.3 & 17.5 \\
\hline & CHD-presymptomatic motor state & 21.9 & 46.0 & 9.3 & 46.5 & 0.4 & 0.0 \\
\hline & Stable-symptomatic motor state & 38.3 & 10.7 & 8.7 & 29.3 & 0.0 & 0.0 \\
\hline \multirow{4}{*}{$\begin{array}{l}\text { Frequency of EMITTED } \\
\text { AFFILIATIVE BEHAVIORS }\end{array}$} & Baseline state & 49.3 & 62.7 & 54.7 & 54.7 & 108.7 & 28.0 \\
\hline & CLD-presymptomatic motor state & 87.6 & 64.2 & 38.9 & 99.7 & 117.3 & 91.0 \\
\hline & CHD-presymptomatic motor state & 84.0 & 30.5 & 31.3 & 76.0 & 90.1 & 44.5 \\
\hline & Stable-symptomatic motor state & 96.3 & 22.7 & 20.3 & 49.0 & 69.7 & 29.0 \\
\hline \multirow{4}{*}{$\begin{array}{l}\text { Frequency of RECEIVED } \\
\text { AFFILIATIVE BEHAVIORS }\end{array}$} & Baseline state & 101.3 & 66.0 & 50.0 & 43.3 & 30.7 & 34.7 \\
\hline & CLD-presymptomatic motor state & 90.5 & 78.7 & 43.8 & 46.1 & 54.5 & 84.3 \\
\hline & CHD-presymptomatic motor state & 77.4 & 52.5 & 42.7 & 51.5 & 27.5 & 42.0 \\
\hline & Stable-symptomatic motor state & 78.0 & 30.7 & 26.7 & 33.0 & 33.3 & 33.0 \\
\hline
\end{tabular}

decreased almost two-fold, whilst no major variation in its frequency of received affiliative behaviors was observed. The four others animals in the social group showed a higher variation in the frequency of social behaviors. Indeed, the frequency of emitted aggressive behaviors in monkey $\mathrm{C}$ decreased almost two-fold, whilst its frequency of received aggressive behaviors decreased about 5 times. Its frequency of emitted submissive behaviors decreased almost three-fold whilst no major variation in its frequency of received submissive behaviors was observed. Finally, monkey $\mathrm{C}$ did not present any variation in its frequency of affiliative behaviors in terms of emission and reception. For monkey $D$, its frequency of emitted aggressive behaviors increased about 7 times and its frequency of received aggressive behaviors decreased almost six-fold. Its frequency of emitted submissive behaviors decreased almost three-fold, whilst its frequency of received submissive behaviors increased about 5 times. Finally, monkey D did not present any variation in its frequency of affiliative behaviors in terms of emission and reception. Monkey E presented a marked decrease of about 162 times in its frequency of emitted aggressive behaviors and did not show any variation in its frequency of received aggressive behaviors. Its frequency of emitted submissive behaviors did not present any variation, whilst its frequency of received submissive behaviors decreased about 88 times. Finally, monkey E did not present any variation in its frequency of emitted affiliative behaviors, whilst its frequency of received affiliative behaviors decreased almost two-fold. The most subordinate animal, monkey $\mathrm{F}$ presented no emission of aggressive behaviors during the CHD-presymptomatic motor state as observed during the baseline state and did not show any major variation in its frequency of received aggressive behaviors. Moreover, its frequency of emitted submissive behaviors did not present any variation, whilst any reception of submissive behaviors was observed as during the baseline state. Finally, monkey F presented a decrease in its frequencies of emitted and received affiliative behaviors almost two-fold.

The dominance hierarchy within the social group was modified during the CHD-presymptomatic motor state $(\mathrm{A}>\mathrm{B}>\mathrm{C}>\mathrm{D}>\mathrm{E}>\mathrm{F})$ compared to the CLD-presymptomatic motor state $(A>B>C>E>F>D)$. Thus, monkey $D$ recovered its initial hierarchical status.

\section{Stable-symptomatic motor state}

A significant difference was observed for the emitted aggressive behaviors during the stable-symptomatic motor state compared 
to the CLD-presymptomatic motor state $[53.5 \pm 32.2$ vs. $11.1 \pm$ 11. $9 ; F_{(3,20)}=3.27, p=0.05$; post-hoc test, $p<0.05$ ] but not the CHD-presymptomatic motor state and the baseline state (Figure 4A). No significant changes were seen in the frequency of emitted and received submissive behaviors, although these frequencies seemed to be lower during the stable-symptomatic motor state than during the three others states (Figure 4B). A significant difference was observed for the emitted affiliative behaviors during the stable-symptomatic motor state compared to the CLD-presymptomatic motor state [83.1 \pm 27.7 vs. $47.8 \pm 30.3 ; F_{(3,20)}=4.02, p<0.05$; post-hoc test, $\left.p<0.05\right]$ but not the $\mathrm{CHD}$-presymptomatic motor state and the baseline state. The frequency of received affiliative behaviors also significantly decreased during the stable-symptomatic motor state compared to the CLD-presymptomatic motor state $[39.1 \pm 19.2$ vs. $66.3 \pm 20.6 ; F_{(3,20)}=5.75, p<0.01$; post-hoc test, $\left.p<0.01\right]$ (Figure 4C).

In subgroup social analyses, there was no significant changes in the frequency of emitted and received aggressive behaviors during the stable-symptomatic motor state compared to the three others states. However, the frequency of emitted aggressive behaviors in dominant animals seemed to be lower during the stable-symptomatic motor state than during the three others states. In subordinate animals, the frequency of aggressive behaviors in terms of emission and reception seemed to be lower during the stable-symptomatic motor state compared to the CLD-presymptomatic motor state (emitted: $8.6 \pm 14.5$ vs. $60.2 \pm$ 47.6; received: $15.7 \pm 4.7$ vs. $62.6 \pm 55.0)$ but not the CHDpresymptomatic motor state and the baseline state (Figure 5A). No significant changes were seen in the frequency of emitted and received submissive behaviors in subgroup social analyses. However, the frequency of emitted submissive behaviors in subordinate animals seemed to be lower during the stable-symptomatic motor state than during the three others states (Figure 5B). No change occurred in the frequency of emitted affiliative behaviors of dominant animals whereas the frequency of received affiliative behaviors was significantly lower during the stable-symptomatic motor state compared to the baseline $[45.11 \pm 28.55$ vs. $72.44 \pm$ 26.27; $F_{(3,8)}=6.33, p<0.05$; post-hoc test, $\left.p<0.05\right]$ and the CLD-presymptomatic motor states $\left[71.0 \pm 24.3 ; F_{(3,8)}=6.33\right.$, $p<0.05$; post-hoc test, $p<0.05$ ]. In subordinate animals, the frequency of affiliative behaviors in terms of emission and reception seemed to be lower during the stable-symptomatic motor state compared to the CLD-presymptomatic motor state (emitted: $49.2 \pm 20.3$ vs. $102.6 \pm 13.4$; received: $33.1 \pm 0.2$ vs. $61.6 \pm$ 20.1) but not the CHD-presymptomatic motor state and the baseline state (Figure 5C).

Individual data (Table 2) showed that a global decrease was observed in the frequency of emission and reception of all social behaviors during the stable-symptomatic motor state compared to the CHD-presymptomatic motor state, except for the most dominant animal. Moreover, any variation of the dominance hierarchy within the social group was observed during the stable-symptomatic motor state compared to the CHDpresymptomatic motor state. Thus, the dominance hierarchy was similar to the dominance hierarchy during the baseline state.

\section{Correlation analyses}

A positive correlation was observed between the frequency of emitted aggressive behaviors and the frequency of received submissive behaviors $(r=0.69, p<0.001)$. A positive correlation was observed between the frequency of emitted aggressive behaviors and the frequency of received affiliative behaviors $(r=0.66, p<0.001)$. A positive correlation was observed between the frequency of received aggressive behaviors and the frequency of emitted submissive behaviors $(r=0.86, p<$ $0.001)$. A negative correlation was observed between the frequency of emitted submissive behaviors and the frequency of received submissive behaviors $(r=-0.41, p<0.05)$. A positive correlation was observed between the frequency of received submissive behaviors and the frequency of received affiliative behaviors $(r=0.77, p<0.001)$. Finally, a negative correlation was observed between the time spent in inactivity and the frequency of emitted aggressive behaviors $(r=-0.51$, $p<0.05)$.

\section{ORDT PERFORMANCE}

The success rate for detour trials was negatively correlated with the clinical scores of animals $(r=-0.80, p<0.001)$ and significantly decreased during the stable-symptomatic state compared to the baseline $[55.4 \pm 11.8 \%$ vs. $81.7 \pm 5.9 \%$; $F_{(3,12)}=10.52, p<0.01$; post-hoc test, $\left.p<0.01\right]$, the CLDpresymptomatic motor $\left[80.4 \pm 6.0 \% ; F_{(3,12)}=10.52, p<\right.$ 0.01 ; post-hoc test, $p<0.01]$ and the CHD-presymptomatic motor states $\left[83.7 \pm 8.4 \% ; F_{(3,12)}=10.52, p<0.01\right.$; post-hoc test, $p<0.01$ ] (Figure 6A). The percentage of errors responses observed in the detour trials was positively correlated with the time spent in inactivity $(r=0.60, p<0.01)$ and significantly increased during the stable-symptomatic state compared to the baseline $\left[40.3 \pm 10.3 \%\right.$ vs. $23.2 \pm 9.8 \% ; F_{(3,12)}=$ 9.83, $p<0.01$; post-hoc test, $p<0.01$ ], the CLD-presymptomatic motor $\left[22.1 \pm 5.0 \% ; F_{(3,12)}=9.83, p<0.01\right.$; post-hoc test, $p<$ $0.01]$ and the CHD-presymptomatic motor states [17.2 $\pm 9.3 \%$; $F_{(3,12)}=9.83, p<0.01$; post-hoc test, $p<0.01$ ] (Figure 6B). Slight changes were seen in the percentage of successes in the easy trials, with a significant decrease in values during the stable-symptomatic motor state compared to the CLDpresymptomatic motor state $[92.1 \pm 4.7 \%$ vs. $97.8 \pm 2.6 \%$; $F_{(3,12)}=4.52, p<0.05$; post-hoc test, $p<0.05$ ] (Figure 6C). Any significant differences between the four states (baseline state and the three motor states) was observed for the percentage of errors in the easy trials (Figure 6D). No significant difference was observed between the two subgroups (Dominant vs. Subordinate).

A negative correlation between the success rate for detour trials and the percentage of errors responses in the detour trials was observed $(r=0.92, p<0.001)$. Similarly, a negative correlation was observed between the success rate for easy trials and the percentage of errors responses in the easy trials $(r=0.93$, $p<0.001)$.

Furthermore, the percentage of errors responses in the detour trials was negatively correlated with the frequency of received affiliative behaviors $(r=-0.52$, $p<0.05)$. 


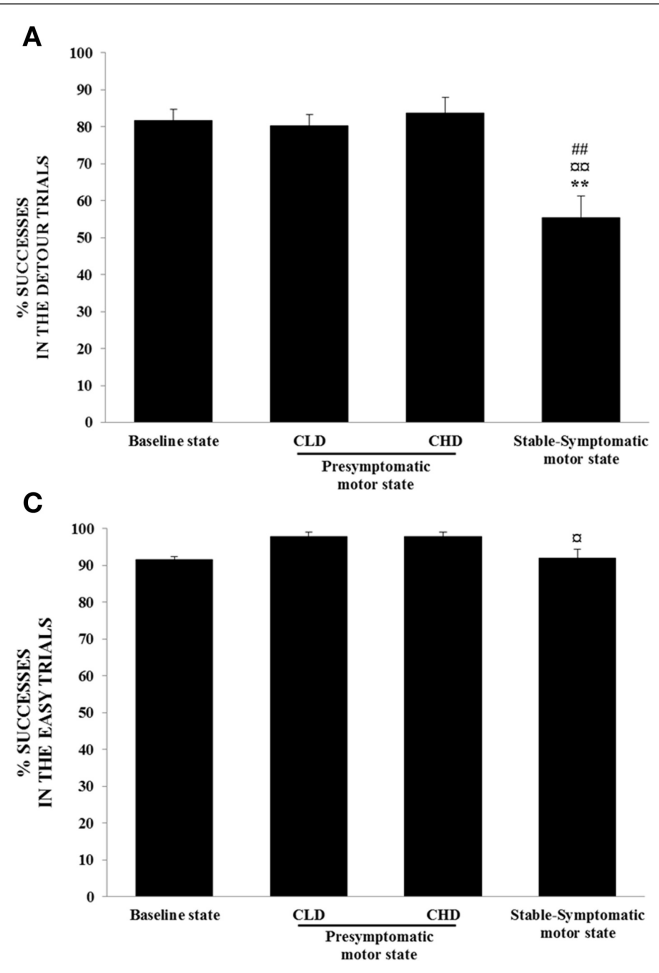

FIGURE 6 | Evolution of ORDT performance from baseline to presymptomatic and symptomatic motor states $(n=4)$. (A) Successes in the detour trials: successes (retrieval of reward on the first reach) were expressed as percentage of total number of detour trials. Values were means $\pm \mathrm{SD}(n=4) .{ }^{* *} p<0.01$ : stable-symptomatic motor state vs. baseline state; ${ }^{a a} p<0.01$ : stable-symptomatic motor state vs. CLD-presymptomatic motor state; ${ }^{\# \#} p<0.01$ : stable-symptomatic motor state vs. CHD-presymptomatic motor state (ANOVA and post-hoc test). (B) Errors in the detour trials: errors (barrier hits, i.e., hitting a transparent side of the box) were expressed as a percentage of responses observed for detour trials. Values were means \pm

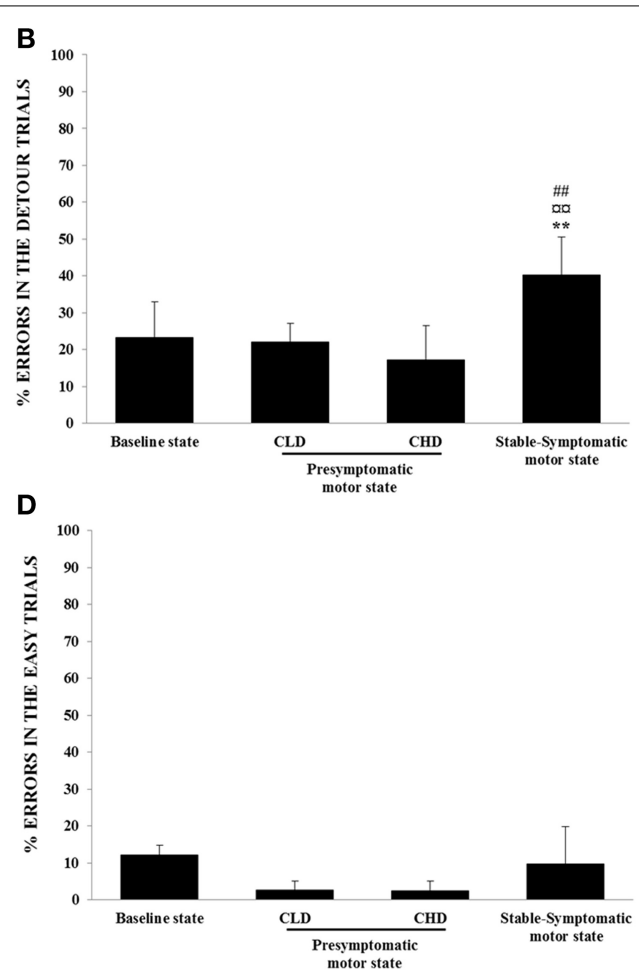

SD $(n=4) .{ }^{* *} p<0.01$ : stable-symptomatic motor state vs. baseline state; ${ }^{a a} p<0.01$ : stable-symptomatic motor state vs. CLD-presymptomatic motor state; ${ }^{\# \#} p<0.01$ : stable-symptomatic motor state vs. CHD-presymptomatic motor state (ANOVA and post-hoc test). (C) Successes in the easy trials: successes (retrieval of reward on the first reach) were expressed as a percentage of the total number of easy trials. Values were means $\pm \mathrm{SD}$ $(n=4) .{ }^{a} p<0.05$ : stable-symptomatic motor state vs. CLD-presymptomatic motor state (ANOVA and post-hoc test). (D) Errors in the easy trials: errors (barrier hits, i.e., hitting a transparent side of the box) were expressed as a percentage of responses observed for easy trials.

\section{${ }^{18}$ F-DOPA UPTAKE \\ Comparisons with the control group}

The comparison of ${ }^{18} \mathrm{~F}$-DOPA uptake ( $\mathrm{Ki}$ value) between the control PET and the CLD PET showed no significant difference, whatever the cerebral area concerned (Figure 7A).

${ }^{18} \mathrm{~F}$-DOPA uptake between the control PET and the CHD PET significantly decreased in the Post $\mathrm{CdN}(3.3 \pm 2.3$ vs. $5.3 \pm 1.5$; random effect models, $z=2.20, p<0.05)$ and the Ins $(1.4 \pm 1.1$ vs. $2.7 \pm 0.6$; random effect models, $z=2.55, p<0.05)$. These results were intensified during the sympto PET compared to the control PET (Post CdN: $0 \pm 0.5$ vs. $5.3 \pm 1.5$; random effect models, $z=5.89, p<0.001$; Ins: $0.4 \pm 0.3$ vs. $2.7 \pm 0.6$; random effect models, $z=4.67, p<0.001$ ) (Figures 7A,B).

During the sympto PET, most regions of the basal ganglia and the cortex showed a significant decrease in ${ }^{18} \mathrm{~F}$-DOPA uptake compared to the control PET. However, a greater decrease was observed in ${ }^{18} \mathrm{~F}$-DOPA uptake in Ant / Post CdN (around 95\%: $94.7 \pm 5.7$ ) than in Ant / Post Put (around 80\%: 80.2 \pm 10.5 ), the V Str (around 55\%: $55.3 \pm 14.6$ ) and the SN (around $38 \%$ : $37.6 \pm 31.6)$. Similarly, a greater decrease in ${ }^{18} \mathrm{~F}$-DOPA uptake was observed in the Ins (around 85\%: $84.8 \pm 11.4$ ) than in the OFC (around 47\%: $47.2 \pm 20.6$ ) and the ACC (around 50\%: $51.4 \pm 37.0$ ). ${ }^{18}$ F-DOPA uptake in the Amyg did not significantly decrease, even during the symptomatic state (Figures 7A,C).

\section{Comparisons within the social group}

The only significant decrease in ${ }^{18} \mathrm{~F}$-DOPA uptake during the CHD PET compared to the CLD PET was observed in the Ins $\left(1.4 \pm 1.2\right.$ vs. $\left.2.3 \pm 0.9 ; C h i^{2}=7.04, p<0.05\right)$ (Figure 7A). This significant decrease intensified during the sympto PET compared to CLD $\left(0.4 \pm 0.3\right.$ vs. $2.3 \pm 0.9 ; C h i^{2}=32.18, p<$ $0.001)$ and CHD PET scans $\left(0.4 \pm 0.3\right.$ vs. $1.4 \pm 1.2 ; \mathrm{Ch}^{2}=$ 9.11, $p<0.01$ ) (Figure 7A). ${ }^{18}$ F-DOPA uptake in the Amyg significantly decreased during the sympto PET compared to CLD PET $\left(1.9 \pm 0.5\right.$ vs. $\left.2.5 \pm 0.7 ; C h i^{2}=5.16, p<0.05\right)$ (Figure 7A).

The comparison of ${ }^{18} \mathrm{~F}$-DOPA uptake between the sympto PET and the CHD PET showed a significant decrease for all cerebral structures except the Amyg. However, in the basal ganglia, a greater decrease in ${ }^{18} \mathrm{~F}$-DOPA uptake was observed in the Ant/Post CdN (around 95\%: 94.2 \pm 7.8 ) than in the Ant/Post 


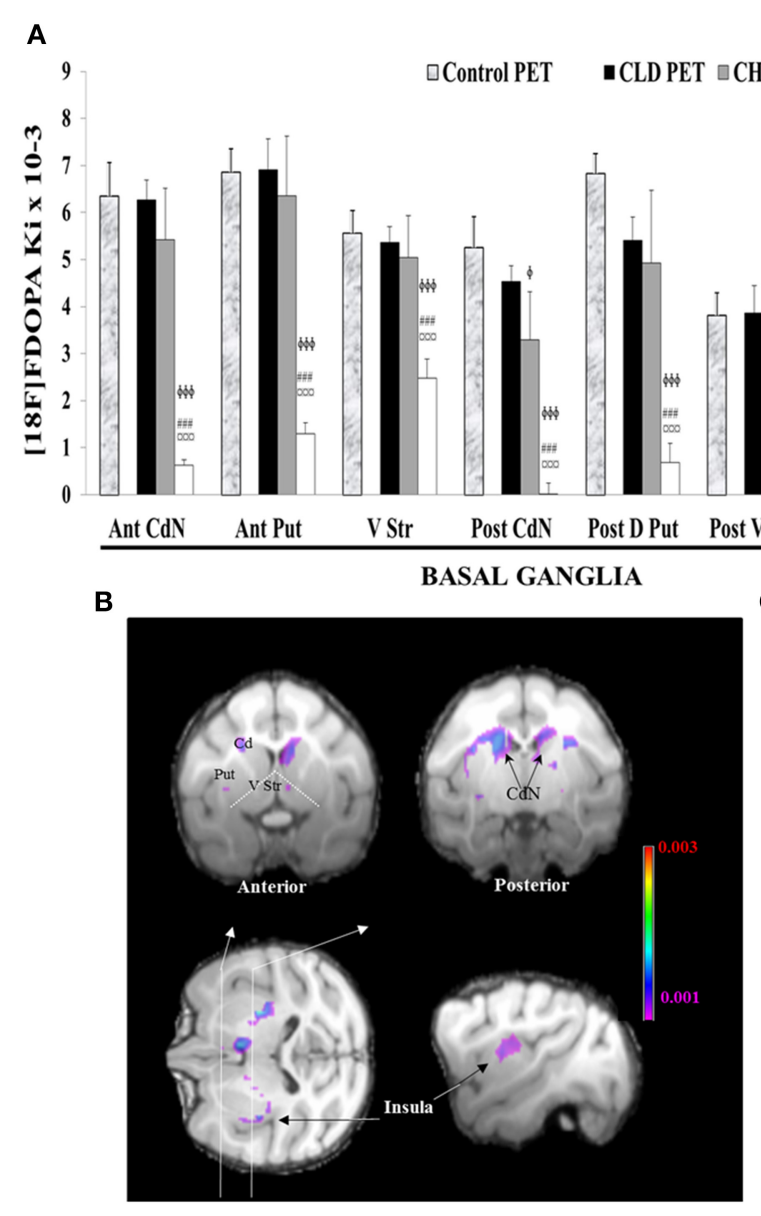

Control - CHD-Presymptomatic
FIGURE 7 | Comparisons of ${ }^{18}$ F-DOPA uptake in PET imaging. (A) The rate of specific uptake (Ki values) was assessed within basal ganglia in relation to ${ }^{18} \mathrm{~F}$-DOPA uptake in the reference brain region (cerebellum). The basal ganglia includes the Anterior and Posterior Caudate Nucleus (Ant CdN/Post CdN), the Anterior Putamen (Ant Put), the Posterior Dorsal and Ventral Putamen (Post DN Put), the Ventral Striatum (V Str) and the Substantia Nigra (SN), as well as uptake in cortical regions such as the Anterior Cingulate Cortex (ACC), the Orbitofrontal Cortex (OFC), the Amygdala (Amyg) and the Insula (Ins). One PET scan was carried out for the control group $(n=5)$ and was considered the Control PET. Three PET scans were carried out for four animals of the social group $(n=4)$ of the study: the first at the end of the presymptomatic motor state following CLD-MPTP protocol (CLD PET), the second during the presymptomatic motor state following CHD-MPTP protocol (CHD PET) period and the third during the
C

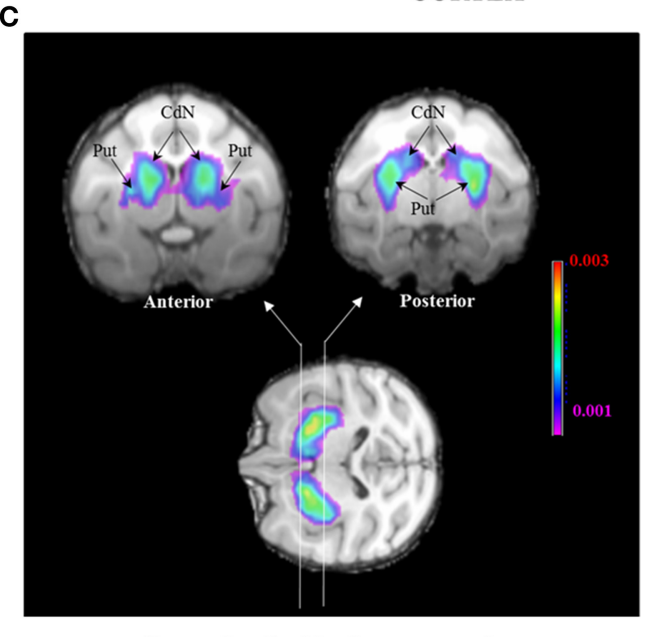

Control - Stable-Symptomatic

symptomatic motor state, when animals developed stable parkinsonian-like motor symptoms ( $\geq 1$ month) (Sympto PET). Comparison with the control group: values of the Control PET scan were means $\pm \operatorname{SD}(n=5)$. Values of the three other PET scans were means $\pm \operatorname{SD}(n=4) .{ }^{\phi \phi \phi} p<0.001,{ }^{\phi \phi} p<0.01$, ${ }^{\phi} p<0.05 ;$ CHD PET, Sympto PET vs. Control PET (random effects models). Comparison within the social group: values of the three PET scans were means \pm SD $(n=4) .{ }^{a a a} p<0.001,{ }^{a} p<0.05$ : CHD PET, Sympto PET vs. CLD PET; \#\# $p<0.001,{ }^{\# \#} p<0.01,{ }^{\#} p<0.05$ : Sympto PET vs. CHD PET (Chi ${ }^{2}$-test). (B) Subtraction images between the average ${ }^{18} \mathrm{~F}$-DOPA uptake of control PET and the average ${ }^{18} \mathrm{~F}$-DOPA uptake of CHD PET. ${ }^{18} \mathrm{~F}$-DOPA uptake decreases in the Post $\mathrm{CdN}$ and the insula were illustrated in frontal sections (black arrows). (C) Subtraction images between the average ${ }^{18} \mathrm{~F}$-DOPA uptake of control PET and the average ${ }^{18} \mathrm{~F}$-DOPA uptake of sympto PET.

putamen (around 75\%: $74.1 \pm 7.4$ ), the V Str (around 50\%: $48.3 \pm 14.1$ ) and the SN (around 25\%: $24.0 \pm 36.2$ ). Similarly, in the cortical areas, a greater decrease of ${ }^{18} \mathrm{~F}$-DOPA uptake was observed in the Ins (around 72\%: $72.5 \pm 24.7$ ) than in the OFC (around 24\%: $23.4 \pm 28.6$ ) and the ACC (around 63\%: $63.1 \pm 25.7)$.

\section{Individual comparisons}

Overall, the results showed a stronger inter-animal variability of the Ki uptake during the CLD and the CHD PET, with the subordinate monkey $\mathrm{D}$ showing the lowest $\mathrm{Ki}$ value in all structures (apart in the ACC and the Ins during the CLD
PET) (Figures 8A,B). ${ }^{18}$ F-DOPA uptake decreased linearly in Post Put, Post CdN and Ins in all three subordinate animals, whereas the Ki uptake in the dominant animal only decreased during the symptomatic PET. A stronger individual variability of Ki was observed in ACC. However, the dynamic of change was comparable between the 4 animals apart for the subordinate monkey $\mathrm{D}$, whose $\mathrm{Ki}$ uptake was not detectable during the sympto PET. Finally, ${ }^{18}$ F-DOPA uptake in V Str and Amyg did not change between CLD and CHD PETs, except for the subordinate monkey $\mathrm{D}$. The only change involving a decrease in ${ }^{18} \mathrm{~F}$-DOPA uptake in $\mathrm{V}$ Str and Amyg occurred during the sympto PET. 


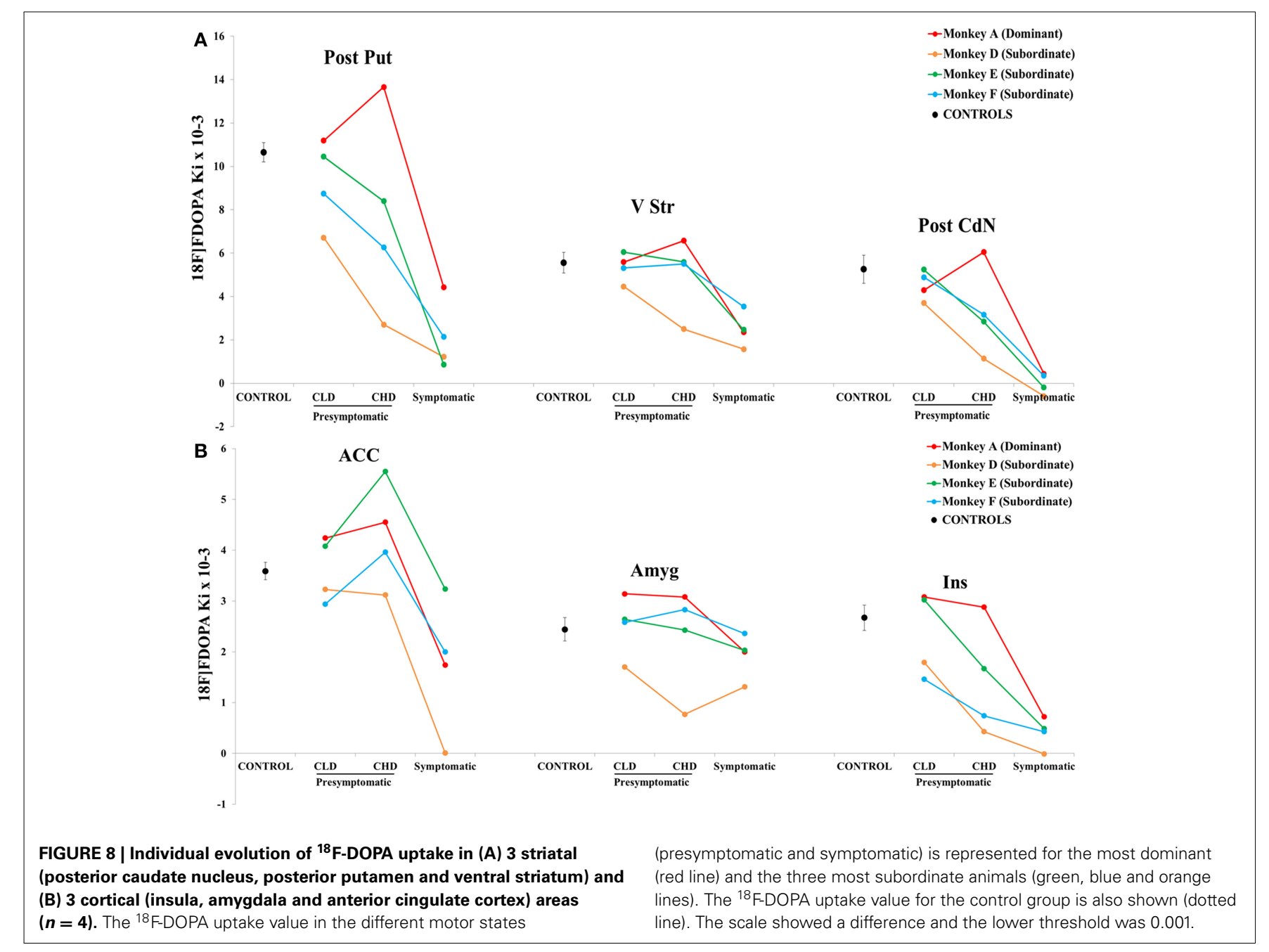

\section{Correlation analyses}

There was a negative correlation between the clinical score and the ${ }^{18} \mathrm{~F}$-DOPA uptake in Ant CdN $(r=-0.88, p<0.001)$, Post $\mathrm{CdN}(r=-0.88, p<001)$, Ant Put $(r=-0.86, p<0.001)$, Post Put $(r=-0.79, p<0.01)$, V Str $(r=-0.75, p<0.01)$, and Ins $(r=-0.74, p<0.01)$. Similarly, there was a negative correlation between the time spent in inactivity and the ${ }^{18} \mathrm{~F}$-DOPA uptake in Ant CdN $(r=-0.88, p<0.05)$, Post CdN $(r=-0.64, p<$ $0.05)$, Ant Put $(r=-0.71, p<0.05)$, Post Put $(r=-0.62, p<$ $0.05)$.

The success rate on detour trials was positively correlated to the ${ }^{18} \mathrm{~F}-\mathrm{DOPA}$ uptake in Ant $\mathrm{CdN}(r=0.77, p<0.05)$, Post CdN $(r=0.74, p<0.05)$, Ant Put $(r=0.79, p<0.05)$, Post Put $(r=$ $0.76, p<0.05)$, ACC $(r=0.77, p<0.05)$ and Ins $(r=0.69$, $p<0.05)$. Moreover, there was a negative correlation between the percentage of errors responses in the detour trials and the ${ }^{18}$ F-DOPA uptake in Ant CdN $(r=0.70, p<0.05)$, Ant Put $(r=0.74, p<0.01)$, Post Put $(r=0.74, p<0.01)$, Ins $(r=$ $0.69, p<0.05)$ and ACC $(r=0.77, p<0.01)$. Finally, a positive correlation was observed between the frequency of emitted affiliative behaviors and the ${ }^{18} \mathrm{~F}-\mathrm{DOPA}$ uptake in Ins $(r=0.67$, $p<0.05)$.

\section{DISCUSSION}

The aim of this study was to focus on social behavioral changes within a group of female long-tailed macaques after chronic administration of low (CLD) and then high (CHD) doses of MPTP. These MPTP administration protocols made it possible to evaluate each individual in three motor states (normal, presymptomatic and symptomatic).

Following the CLD-MPTP protocol, a significant increase was highlighted in the frequency of aggressive and affiliative behaviors from the baseline state to the CLD-presymptomatic motor state. Indeed, a higher number of conflicts followed by reconciliation events were observed. More precisely, these conflicts occurring during the CLD-MPTP protocol were very intense with slapping, biting and other aggressive behaviors involving direct contact between individuals. None of these aggressive behaviors were observed in the baseline state, where the few conflicts observed were of low intensity and mainly limited to visual threats with the extensive use of facial expressions. Interestingly, these social behavioral changes were only shown by the subordinate animals. Moreover, a temporary change of the dominance hierarchy was observed; thus, monkey D presented the lowest hierarchical status compared to monkeys $\mathrm{E}$ and $\mathrm{F}$. Individual data within this 
subgroup were too heterogeneous to show any significant variations in the three categories of social behaviors. Indeed, only the frequency of emitted affiliative behaviors increased significantly in the CLD-presymptomatic motor state compared to the baseline state. A clearer picture could be obtained by increasing the number of animals in this study, taking the hierarchical status into consideration.

From the CLD-presymptomatic to the CHD-presymptomatic motor states, the frequency of social behaviors decreased. The dominance hierarchy was similar to the baseline state; thus monkey D recovered its initial hierarchical status. At this time, quantitative data analysis in PET scans made it possible to highlight a dopaminergic denervation in two brain structures, the insula and the posterior caudate nucleus, which could therefore be involved in the social behavioral changes observed from the CLDpresymptomatic motor state to the $\mathrm{CHD}$-presymptomatic motor state.

Finally, during the symptomatic motor state, the frequency of social behaviors was lower across all categories, than the frequency observed in the CLD and the CHD-presymptomatic motor states. The dominance hierarchy was not modified and was similar to that observed during the baseline state. Motor and cognitive disorders were also observed in this stable-symptomatic state. PET scans results then showed a dopaminergic denervation in all the evaluated cortical and subcortical structures.

In the present study, the choice was made to intoxicate not just one animal with MPTP, but all the animals in the group. This decision was made in order to increase the probability of observing social behavioral changes due to inter-individual variability of sensitivity to MPTP in monkeys. Moreover, this study did not use a control group to compare the distribution of interactions over time in the present study group. This is explained by the fact that the stability of primate groups can vary despite similar sizes and similar ecological conditions (Sueur et al., 2011) and the distribution of grooming can differ considerably between groups (Perry, 1996; Manson et al., 1999; Dufour et al., 2011). Consequently, it is more efficient to analyze social relationships of a single group over time when investigating network stability or instability. For example, this enables us to compare different observation periods where breeding conditions have changed, and study how individuals within one same group cope with perturbation (Dufour et al., 2011). The present study examines the evolution of social interactions over time in order to understand how our group of female macaques coped socially with disease-related changes.

\section{CHRONIC LOW- AND HIGH-DOSE PROTOCOL IS EFFECTIVE TO OBSERVE SOCIAL BEHAVIORAL CHANGES AND DISSOCIATE THEM FROM COGNITIVE AND MOTOR IMPAIRMENTS}

This is the first study describing social behavioral changes during the presymptomatic motor state following MPTP administration before the onset of cognitive and motor disorders. On the one hand, a previous study has also shown social behaviors changes (aggressive and affiliative) during the symptomatic motor state induced by MPTP administration (hemiparkinsonian model in vervet monkey model) (Melega et al., 1996). However, this study was performed during the symptomatic motor state. Thus, it is difficult to dissociate the effect of motor disorders on social behavioral changes. On the other hand, it is important to note that two previous studies in the CLD-MPTP-treated macaque model showed cognitive impairment in the presymptomatic motor state (Schneider and Pope-Coleman, 1995; Vezoli et al., 2011), which was not the case in the present study. Indeed, cognitive and motor disturbances appeared only during the symptomatic motor state following the CHD-MPTP proto$\mathrm{col}$, and their occurrence was almost simultaneous. Thus, it was difficult to really dissociate the impact of motor disorders on cognitive abilities. Several hypotheses could explain the differences in cognition results between this study and the two studies mentioned above. Firstly, the dose of MPTP administered to animals was different: $0.1 \mathrm{mg} / \mathrm{kg}$ in the present study vs. $0.05-$ $0.075 \mathrm{mg} / \mathrm{kg}$ in Schneider's study and $0.2 \mathrm{mg} / \mathrm{kg}$ in Vezoli's study. Secondly, MPTP was administered every $4-5$ days for more than 50 weeks in the present study vs. 2-3 times per week for 24 weeks in Schneider's study and every 3-4 days for 5-25 weeks in Vezoli's study. Thirdly, the social housing could also have an impact (Prescott et al., 2010), as a possible protective effect against development of cognitive deficits. Finally, the sex and age of animals differ between studies (Prescott et al., 2010; Darusman et al., 2014). Moreover, over-training might have an impact on such simple task as it was shown to be associated with less engagement of cognitive control areas, which is specifically tested with this task (Patel et al., 2013). In the present study, there was an apparent gain in performance in CLD-presymptomatic motor state compared to baseline state; this might indicate that animals maintained learning capacities on the task with the MPTP regimen used and the task chosen in the present study.

\section{HETEROGENEITY OF SOCIAL BEHAVIORAL CHANGES ACCORDING TO HIERARCHICAL STATUS}

In the present study, social behavioral changes were only observed in the subordinate subgroup when compared to the dominant subgroup. It was also important to note that the most dominant individual was also involved in post-conflicts reconciliation events, suggesting that it retained its regulatory role within the social group (Petit and Thierry, 1992).

Hierarchical status was assessed using methods that have been validated and widely used in ethology (David, 1987, 1988; Kaplan et al., 2002). Several studies have already demonstrated the specific role played by dopamine in hierarchical status even if other neurotransmitters such as noradrenalin and serotonin have been implicated in behavioral disorders (Raleigh et al., 1991; Siever, 2008; Krämer et al., 2011). A study by Kaplan et al. (Kaplan et al., 2002) has shown that in non-human primates, a higher concentration of homovanillic acid (dopamine's catabolite) was found in the cerebrospinal fluid of dominant animals than in subordinate animals, whatever their gender. PET imaging studies with a specific $D_{2} / D_{3}$ receptor radiotracer also highlighted differences in radiotracer uptake according to hierarchical status in male long-tailed monkeys (Morgan et al., 2002; Nader et al., 2012) and in humans (Martinez et al., 2010). Interestingly, levels of $\mathrm{D}_{2} / \mathrm{D}_{3}$ receptor availability appeared to be sensitive to changes in housing conditions. This sensitivity was such that the transition from individual to social housing resulted in significant increases in $\mathrm{D}_{2} / \mathrm{D}_{3}$ levels in dominant male animals, whilst subordinate 
animals showed no change (Morgan et al., 2002). Nader et al. recently extended their earlier work in male long-tailed monkeys to female long-tailed monkeys, with the additional use of a dopamine transporter (DAT) radiotracer (Nader et al., 2012). Their results showed that although neither DAT nor $\mathrm{D}_{2} / \mathrm{D}_{3}$ receptor availability in the caudate nucleus and putamen was predictive of social rank, both significantly changed after the formation of social hierarchies in female long-tailed monkeys. Dopamine $\mathrm{D}_{2} / \mathrm{D}_{3}$ receptor availability significantly increased in females that became dominant, whereas DAT availability decreased in subordinate females. Otherwise, an association between the dopamine $\mathrm{D}_{4}$ receptor and a personality trait such as novelty seeking has been demonstrated in both humans (Benjamin et al., 1996; Ebstein et al., 1996) and non-human primates (Bailey et al., 2007). In the present study, the role of dopamine in the behavioral changes observed can be evaluated through the use of ${ }^{18} \mathrm{~F}$-Dopa radiotracer, a specific radiotracer of dopaminergic innervation, during PET scans.

\section{A POSSIBLE ASSOCIATION BETWEEN DOPAMINERGIC DENERVATION AND SOCIAL BEHAVIORAL CHANGES}

During the CLD-presymptomatic motor state following the CLDMPTP protocol, whereas a significant increase of the frequency of social behaviors compared to the baseline state was observed, no significant difference was identified between the Ki values of ${ }^{18} \mathrm{~F}$ Dopa uptake in the MPTP-treated animals compared to that of the control animals. However, as MPTP-treated animals were not their own control, it was impossible to know if they really present any variation of their Ki values of ${ }^{18} \mathrm{~F}$-Dopa uptake. Nevertheless, it was interesting to note that two subordinate animals (monkeys $\mathrm{D}$ and $\mathrm{F}$ ) had lower $\mathrm{Ki}$ uptake values in the insula and dorsal putamen than those observed in control individuals. Recent studies have shown that neural circuits (Noonan et al., 2014) and dopaminergic innervation (Nader et al., 2012) could differ from dominant to subordinate individuals. Thus, a brain structure with initially low Ki values would probably be more sensitive to MPTP intoxication and would therefore show dopaminergic denervation earlier than other brain structures. However, MPTP sensitivity was not seen to differ according to hierarchical status in this study. Finally, the use of non-dopaminergic radiotracer, including namely serotoninergic radiotracers, would have probably shown interesting and complementary results to the present study (Raleigh et al., 1991; Siever, 2008; Krämer et al., 2011). Indeed, a study in male vervet monkeys has shown that there was a distinction between dominance and aggression and has strongly suggested that when hierarchical relationships are uncertain, serotonergic mechanisms may mediate the behaviors which permit a male to attain high dominance status (Raleigh et al., 1991). Moreover, whereas previous reports have suggested an inverse relationship between serotonin level and aggressive behavior with low levels of serotonin leading to higher aggression and vice versa, such a simple relationship seemed to be inconsistent with the current data obtained in the study Krämer et al. (2011).

During the CHD-presymptomatic motor state following the CHD-MPTP protocol, a significant decrease of the frequency of social behaviors compared to the CLD-presymptomatic motor state was observed. Moreover, a dopaminergic denervation in the insula and the posterior caudate was identified, only in the three subordinate animals but not in the dominant animal.

The insula and the caudate nucleus are both involved in the organization of the social behavioral network or "social brain network," which also includes the prefrontal cortex, the amygdala, the hippocampus, the medial preoptic area, the hypothalamus, the anterior cingulate cortex and the basal ganglia as a whole (Newman, 1999; Skuse and Gallagher, 2009). This network is also closely linked to the reward circuit (O'Connell and Hofmann, 2011). The two abovementioned networks are involved in the regulation of emotions and aggression (Davidson et al., 2000; Siever, 2008).

More specifically, the insula plays a role in the regulation and expression of several emotions, including disgust (Lamm and Singer, 2010; Jezzini et al., 2012), an emotion that is particularly impaired in parkinsonian patients (Suzuki et al., 2006), as well as the expression of social behavior. A recent study in rhesus macaques used intracortical microstimulations in different regions of the insula highlight its involvement in the expression of disgust and in the expression of an essential affiliative facial expression in social relationships in macaques, the "lipsmacking display" (Caruana et al., 2011). Thus, an impairment of the insula during the CHD-presymptomatic motor state could be associated with the impaired expression of affiliative behaviors, as suggested by the positive correlation observed in our study between the frequency of emitted affiliative behaviors and the ${ }^{18} \mathrm{~F}$-DOPA uptake in the insula.

The caudate nucleus is particularly involved in the human "trust network" (Baumgartner et al., 2008), and its activity increases throughout cooperation between protagonists (De Dreu, 2012). An impairment of the caudate nucleus during the presymptomatic motor state could therefore be associated with an impairment of social relationships. This would explain why the frequency of social behaviors decreased from the CLD to the CHD presymptomatic motor states. This decrease could be associated with a "loss of emotional interest," and could also correspond to an "apathetic state" during the presymptomatic motor state. Indeed, apathy is characterized in humans by a loss of interest and a lower participation in usual activities, a lack of initiative, diminished initiated activities, an emotional indifference and a flat affect. Moreover, apathy can be described in 3 dimensions (Levy and Dubois, 2006): cognitive, emotional and behavioral. However, the lack of effect on the ORDT performance during the CHD-presymptomatic motor state excluded the cognitive dimension (Brown et al., 2012) and argued to possible emotional and/or behavioral attempt.

Finally, and surprisingly, other cortical structures belonging to the social behavior network, including the orbitofrontal cortex, the anterior cingulate cortex, amygdala, and a limbic structure of the basal ganglia, the ventral striatum, did not seem to be involved in behavioral changes observed during the presymptomatic motor states. Indeed, these structures were only impaired during the stable-symptomatic motor state and could be associated not only with social behavioral changes but also with cognitive and motor disorders, as suggested by the correlations observed between the Ki value of several cerebral structures and motor/cognitive disorders. This suggests that the dopaminergic 
innervations of these brain structures are less sensitive to MPTP in our experimental conditions.

\section{CONCLUSION}

In conclusion, the present study of the MPTP-treated non-human primate model of Parkinson's disease showed that behavioral changes appeared early, before the onset of motor and cognitive disorders. This could be compared with clinical data obtained in parkinsonian patients showing a high incidence of behavioral changes in the early stages of the disease (Aarsland et al., 2009). Furthermore, these behavioral changes were most common among subordinate individuals. Finally, the results of the present study revealed the early sensitivity of cortical structures such as the insula to MPTP. However, additional studies including more animals to limit the inter-individual variability would be necessary to clarify the role of hierarchical status on behavioral changes. Likewise, the use of non-dopaminergic radiotracer, including namely serotoninergic radiotracers, would have probably shown interesting and complementary results to the present study.

\section{ACKNOWLEDGMENT}

The authors are very grateful to Nicolas Costes and Didier Le Bars for PET acquisitions.

\section{SUPPLEMENTARY MATERIAL}

The Supplementary Material for this article can be found online at: http://www.frontiersin.org/journal/10.3389/fnbeh.2015. 00042/abstract

\section{REFERENCES}

Aarsland, D., Marsh, L., and Schrag, A. (2009). Neuropsychiatric symptoms in Parkinson's disease. Mov. Disord. 24, 2175-2186. doi: 10.1002/mds.22589

Adams, M. R., Kaplan, J. R., and Koritnik, D. R. (1985). Psychosocial influences on ovarian endocrine and ovulatory function in Macaca fascicularis. Physiol. Behav. 35, 935-940. doi: 10.1016/0031-9384(85)90262-8

Altmann, J. (1974). Observational study of behavior: sampling methods. Behaviour 49, 227-267. doi: 10.1163/156853974X00534

Amaral, D. G. (2002). The primate amygdala and the neurobiology of social behavior: implications for understanding social anxiety. Biol. Psychiatr. 51, 11-17. doi: 10.1016/S0006-3223(01)01307-5

Angst, W. (1975). "Basic data and concepts on the social organization of Macaca Fascicularis," in Primate Behavior, ed L. A. Rosenblum (New York, NY: Academic Press), 325-388.

Bailey, J. N., Breidenthal, S. E., Jorgensen, M. J., McCracken, J. T., and Fairbanks, L. A. (2007). The association of DRD4 and novelty seeking is found in a nonhuman primate model. Psychiatr. Genet. 17, 23-27. doi: 10.1097/YPG.0b013e32801140f2

Ballanger, B., Tremblay, L., Sgambato-Faure, V., Beaudoin-Gobert, M., Lavenne, F., Le Bars, D., et al. (2013). A multi-atlas based method for automated anatomical Macaca fascicularis brain MRI segmentation and PET kinetic extraction. Neuroimage 77, 26-43. doi: 10.1016/j.neuroimage.2013.03.029

Barraud, Q., Lambrecq, V., Forni, C., McGuire, S., Hill, M., Bioulac, B., et al. (2009). Sleep disorders in Parkinson's disease: the contribution of the MPTP non-human primate model. Exp. Neurol. 219, 574-582. doi: 10.1016/j.expneurol.2009.07.019

Baumgartner, T., Heinrichs, M., Vonlanthen, A., Fischbacher, U., and Fehr, E. (2008). Oxytocin shapes the neural circuitry if trust and trust adaptation in humans. Neuron 58, 639-650. doi: 10.1016/j.neuron.2008.04.009

Benjamin, J., Li, L., Patterson, C., Greenberg, B. D., Murphy, D. L., and Hamer, D. H. (1996). Population and familial association between the D4 dopamine receptor gene and measures of Novelty Seeking. Nat. Genet. 12, 81-84. doi: 10.1038/ng0196-81
Bentley-Condit, V. K., and Smith, E. O. (1999). Female dominance and female social relationships among yellow baboons (Papio hamadryas cynocephalus). Am. J. Primatol. 47, 321-334.

Blesa, J., Pifl, C., Sánchez-González, M. A., Juri, C., García-Cabezas, M. A., Adánez, R., et al. (2012). The nigrostriatal system in the presymptomatic and symptomatic stages in the MPTP monkey model: a PET, histological and biochemical study. Neurobiol. Dis. 48, 79-91. doi: 10.1016/j.nbd.2012.05.018

Brent, L., and Veira, Y. (2002). Social behavior of captive Indochinese and Insular long-tailed macaques (Macaca fascicularis) following transfer to a new facility. Int. J. Primatol. 23, 147-159. doi: 10.1023/A:1013206125884

Brown, C. A., Campbell, M. C., Karimi, M., Tabbal, S. D., Loftin, S. K., Tian, L. L., et al. (2012). Dopamine pathway loss in nucleus accumbens and ventral tegmental area predicts apathetic behavior in MPTP-lesioned monkeys. Exp. Neurol. 236, 190-197. doi: 10.1016/j.expneurol.2012.04.025

Camus, S. M. J., Blois-Heulin, C., Li, Q., Hausberger, M., and Bezard, E. (2013). Behavioural profiles in captive-bred cynomolgus macaques: towards monkey models of mental disorders? PLoS ONE 8:e62141. doi: 10.1371/journal.pone.0062141

Caruana, F., Jezzini, A., Sbriscia-Fioretti, B., Rizzolatti, G., and Gallese, V. (2011). Emotional and social behaviors elicited by electrical stimulation of the insula in the macaque monkey. Curr. Biol. 21, 195-199. doi: 10.1016/j.cub.2010.12.042

Chassain, C., Eschalier, A., and Durif, F. (2001). Assessment of motor behavior using a video system and a clinical rating scale in parkinsonian monkeys lesioned by MPTP. J. Neurosci. Met. 111, 9-16. doi: 10.1016/S01650270(01)00425-3

Chaumette, T., Derkinderen, P., Bruley des Varannes, S., Bezard, E., and Accary, D. (2009). Neurochemical plasticity in the enteric nervous system of a primate animal model of experimental Parkinsonism. Neurogastroenterol. Motil. 21, 215-222. doi: 10.1111/j.1365-2982.2008.01226.x

Costes, N., Zimmer, L., Reilhac, A., Lavenne, F., Ryvlin, P., and Le Bars, D. (2007). Test-retest reproducibility of 18 F-MPPF PET in healthy humans: a reliability study. J. Nucl. Med. 48, 1279-1288. doi: 10.2967/jnumed.107.041905

Czoty, P. W., Riddick, N. V., Gage, H. D., Sandridge, M., Nader, S. H., Garg, S., et al. (2009). Effect of menstrual cycle phase on dopamine D2 receptor availability in female cynomolgus monkeys. Neuropsychopharmacology 34, 548-554. doi: 10.1038/npp.2008.3

Darusman, H. S., Call, J., Sajuthi, D., Schapiro, S. J., Gjedde, A., Kalliokoski, O., et al. (2014). Delayed response task performance as a function of age in cynomolgus monkeys (Macaca fascicularis). Primates 55, 259-267. doi: 10.1007/s10329-0130397-8

David, H. A. (1987). Ranking from unbalanced paired-comparison data. Biometrika 74, 432-436. doi: 10.1093/biomet/74.2.432

David, H. A. (1988). The Method of Paired Comparisons. London: Charles Griffin.

Davidson, R. J., Putnam, K. M., and Larson, C. L. (2000). Dysfunction in the neural circuitry of emotion regulation-a possible prelude to violence. Science 289 , 591-594. doi: 10.1126/science.289.5479.591

Decamp, E., and Schneider, J. S. (2004). Attention and executive function deficits in chronic low-dose MPTP-treated non-human primates. Eur. J. Neurosci. 20, 1371-1378. doi: 10.1111/j.1460-9568.2004.03586.x

De Dreu, C. K. (2012). Oxytocin modulates cooperation within and competition between groups: an integrative review and research agenda. Horm. Behav. 61, 419-428. doi: 10.1016/j.yhbeh.2011.12.009

Dufour, V., Sueur, C., Whiten, A., and Buchanan-Smith, H. M. (2011). The impact of moving to a novel environment on social networks, activity and wellbeing in two New World primates. Am. J. Primatol. 73, 802-811. doi: 10.1002/ajp.20943

Ebstein, R. P., Novick, O., Umansky, R., Priel, B., Osher, Y., Blaine, D., et al. (1996). Dopamine D4 receptor (D4DR) Exon III polymorphism associated with the human personality trait of Novelty Seeking. Nat. Genet. 12, 78-80. doi: 10.1038/ng0196-78

Gallagher, D. A., and Schrag, A. (2012). Psychosis, apathy, depression and anxiety in Parkinson's disease. Neurobiol. Dis. 46, 581-589. doi: 10.1016/j.nbd.2011.12.041

Gammell, M. P., De Vries, H., Jennings, D. J., Carlin, C. M., and Hayden, T. J. (2003). David's score: a more appropriate dominance ranking method than Clutton-Brock et al's index. Anim. Behav. 66, 601-605. doi: 10.1006/anbe.2003.2226

Gibb, W. R., and Lees, A. J. (1991). Anatomy, pigmentation, ventral and dorsal subpopulations of the substantia nigra, and differential cell death in Parkinson's disease. J. Neurol. Neurosurg. Psychiatry 54, 388-396. doi: 10.1136/jnnp. 54.5.388 
Gomez-Mancilla, B., Boucher, R., Gagnon, C., Di Paolo, T., Markstein, R., and Bédard, P. J. (1993). Effect of adding the D1 agonist CY 208-243 to chronic bromocriptine treatment. I: evaluation of motor parameters in relation to striatal catecholamine content and dopamine receptors. Mov. Disord. 8, 144-150. doi: $10.1002 / \mathrm{mds} .870080205$

Gumert, M. D. (2010). "Dominance and reciprocity in the grooming relationships of female long-tailed macaques (Macaca fascicularis) in Indonesia," in Indonesian Primates (Developments in Primatology: Progress and Prospects), eds S. Gursky-Doyen and J. Supriatna (Springer Science+Business Media) Chapter 18, 309-341.

Haber, S. N., and Knutson, B. (2010). The reward circuit: linking primate anatomy and human imaging. Neuropsychopharmacology 35, 4-26. doi: 10.1038/npp.2009.129

Hirsch, E. C., Orieux, G., Muriel, M. P., Francois, C., and Feger, J. (2003). Nondopaminergic neurons in Parkinson's disease. Adv. Neurol. 91, 29-37.

Isley, M. (2010). Implanon: the subdermal contraceptive implant. J. Pediatr. Adolesc. Gynecol. 23, 364-367. doi: 10.1016/j.jpag.2010.03.006

Jenner, P. (2003). The MPTP-treated primate as a model of motor complications in PD: primate model of motor complications. Neurology 61, S4-S11. doi: 10.1212/WNL.61.6_suppl_3.S4

Jezzini, A., Caruana, F., Stoianov, I., Gallese, V., and Rizzolatti, G. (2012). Functional organization of the insula and inner perisylvian regions. Proc. Natl. Acad. Sci. U.S.A. 109, 10077-10082. doi: 10.1073/pnas.1200 143109

Kaplan, J. R., Manuck, S. B., Fontenot, M. B., and Mann, J. J. (2002). Central nervous system monoamine correlates of social dominance in cynomolgus monkeys (Macaca fascicularis). Neuropsychopharmacology 26, 431-443. doi: 10.1016/S0893-133X(01)00344-X

Kish, S. J., Shannak, K., and Hornykiewicz, O. (1988). Uneven pattern of dopamine loss in the striatum of patients with idiopathic Parkinson's disease. Pathophysiologic and clinical implications. N. Engl. J. Med. 318, 876-880. doi: 10.1056/NEJM198804073181402

Kish, S. J., Tong, J., Hornykiewicz, O., Rajput, A., Chang, L. J., Guttman, M., et al. (2008). Preferential loss of serotonin markers in caudate versus putamen in Parkinson's disease. Brain 131, 120-131. doi: 10.1093/brain/ awm239

Kortekaas, R., Eshuis, S. A., Andringa, G., Cools, A. R., and Leenders, K. L. (2013). Motor behavior correlates with striatal [18F]-DOPA uptake in MPTPlesioned primates. Neurochem. Int. 62, 349-353. doi: 10.1016/j.neuint.2013. 01.021

Krämer, U. M., Riba, J., Richter, S., and Münte, T. F. (2011). An fMRI study on the role of serotonin in reactive aggression. PLOS ONE 6:e27668. doi: 10.1371/journal.pone. 0027668

Lamm, C., and Singer, T. (2010). The role of anterior insular cortex in social emotions. Brain Struct. Funct. 214, 579-591. doi: 10.1007/s00429-010-0251-3

Levy, R., and Dubois, B. (2006). Apathy and the functional anatomy of the prefrontal cortex-basal ganglia circuits. Cereb. Cortex 16, 916-928. doi: 10.1093/cercor/bhj043

Machado, C. J., and Bachevalier, J. (2006). The impact of selective amygdala, orbital frontal cortex, or hippocampal formation lesions on established social relationships in rhesus monkeys (Macaca mulatta). Behav. Neurosci. 120, 761-786. doi: 10.1037/0735-7044.120.4.761

Machado, C. J., Kazama, A. M., and Bachevalier, J. (2009). Impact of amygdala, orbital frontal, or hippocampal lesions on threat avoidance and emotional reactivity in nonhuman primates. Emotion 9, 147-163. doi: 10.1037/a0014539

Madras, B. K., Fahey, M. A., Goulet, M., Lin, Z., Bendor, J., Goodrich, C., et al. (2006). Dopamine transporter (DAT) inhibitors alleviate specific parkinsonian deficits in monkeys: association with DAT occupancy in vivo. J. Pharmacol. Exp. Ther. 319, 570-585. doi: 10.1124/jpet.106.105312

Manson, J. H., Rose, L., Perry, S., and Gros-Louis, J. (1999). Dynamics of femalefemale social relationships in wild Cebus capucinus: data from two Costa Rican sites. Int. J. Primatol. 20, 679-706. doi: 10.1023/A:1020700718458

Martinez, D., Orlowska, D., Narendran, R., Slifstein, M., Liu, F., Kumar, D., et al. (2010). Dopamine type $2 / 3$ receptor availability in the striatum and social status in human volunteers. Biol. Psychiatr. 67, 275-278. doi: 10.1016/j.biopsych.2009.07.037

Martínez-Martín, P., and Damián, J. (2010). Parkinson disease: depression and anxiety in Parkinson disease. Nat. Rev. Neurol. 6, 243-245. doi: 10.1038/nrneurol.2010.49
Melega, W. P., Raleigh, M. J., Stout, D. B., DeSalles, A. A., Cherry, S. R., Blurton-Jones, M., et al. (1996). Longitudinal behavioral and 6-[18F]fluoro-LDOPA-PET assessment in MPTP-hemiparkinsonian monkeys. Exp. Neurol. 141, 318-329. doi: 10.1006/exnr.1996.0167

Michael, R. P., and Zumpe, D. (1993). A review of hormonal factors influencing the sexual and aggressive behavior of macaques. Am. J. Primatol. 30, 213-241. doi: 10.1002/ajp.1350300306

Morgan, D., Grant, K. A., Gage, H. D., Mach, R. H., Kaplan, J. R., Prioleau, O. A., et al. (2002). Social dominance in monkeys: dopamine D2 receptors and cocaine self-administration. Nat. Neurosci. 5, 169-174. doi: 10.1038/nn798

Morgan, D., Grant, K. A., Prioleau, O. A., Nader, S. H., Kaplan, J. R., and Nader, M. A. (2000). Predictors of social status in cynomolgus monkeys (Macaca fascicularis) after group formation. Am. J. Primatol. 52, 115-131. doi: 10.1002/1098-2345(200011)52:3<115::AID-AJP1>3.0.CO;2-Z

Nader, M. A., Nader, S. H., Czoty, P. W., Riddick, N. V., Gage, H. D., Gould, R. W., et al. (2012). Social dominance in female monkeys: dopamine receptor function and cocaine reinforcement. Biol. Psychiatr. 72, 414-421. doi: 10.1016/j.biopsych.2012.03.002

Neumane, S., Mounayar, S., Jan, C., Epinat, J., Ballanger, B., Costes, N., et al. (2012). Effects of dopamine and serotonin antagonist injections into the striatopallidal complex of asymptomatic MPTP-treated monkeys. Neurobiol. Dis. 48, 27-39. doi: 10.1016/j.nbd.2012.06.002

Newman, S. W. (1999). The medial extended amygdala in male reproductive behavior. A node in the mammalian social behavior network. Ann. N.Y. Acad. Sci. 877, 242-257. doi: 10.1111/j.1749-6632.1999.tb09271.x

Noonan, M. P., Sallet, J., Mars, R. B., Neubert, F. X., O’Reilly, J. X., Andersson, J. L., et al. (2014). A neural circuit covarying with social hierarchy in macaque. PLoS Biol. 12:e1001940. doi: 10.1371/journal.pbio.1001940

O'Connell, L. A., and Hofmann, H. A. (2011). The vertebrate mesolimbic reward system and social behavior network: a comparative synthesis. J. Comp. Neurol. 519, 3599-3639. doi: 10.1002/cne.22735

Palfi, S., Ferrante, R. J., Brouillet, E., Beal, M. F., Dolan, R., Guyot, M. C., et al. (1996). Chronic 3-nitropropionic acid treatment in baboons replicates the cognitive and motor deficits of Huntington's disease. J. Neurosci. 16, 3019-3025.

Patel, R., Spreng, R. N., and Turner, G. R. (2013). Functional brain changes following cognitive and motor skills training. A quantitative meta-analysis. Neurorehab. Neural Repair 27, 187-199. doi: 10.1177/1545968312461718

Patlak, C. S., Blasberg, R. G., and Fenstermacher, J. D. (1983). Graphical evaluation of blood-to-brain transfer constants from multiple-time uptake data. J. Cereb. Blood Flow Metab. 3, 1-7. doi: 10.1038/jcbfm.1983.1

Pedersen, K. F., Alves, G., Aarsland, D., and Larsen, J. P. (2009). Occurrence and risk factors for apathy in Parkinson disease: a 4-year prospective longitudinal study. J. Neurol. Neurosurg. Psychiatr. 80, 1279-1282. doi: 10.1136/jnnp.2008.170043

Pell, M. D., Cheang, H. S., and Leonard, C. L. (2006). The impact of Parkinson's disease on vocal-prosodic communication from the perspective of listeners. Brain Lang. 97, 123-134. doi: 10.1016/j.bandl.2005.08.010

Perez-Otano, I., Oset, C., Luquin, M. R., Herrero, M. T., Obeso, J. A., and Del Rio, J. (1994). MPTP-induced parkinsonism in primates: pattern of striatal dopamine loss following acute and chronic administration. Neurosci. Lett. 175, 121-125. doi: 10.1016/0304-3940(94)91094-4

Perry, S. (1996). Female-female relationships in wild white-faced capuchin monkeys, Cebus capucinus. Am. J. Primatol. 40, 167-182.

Petit, O., and Thierry, B. (1992). Affiliative function of the silent bared-teeth display in moor macaques (Macaca maurus): further evidence for the particular status of Sulawesi macaques. Int. J. Primatol. 13, 97-105. doi: 10.1007/BF02547729

Prescott, M. J., Brown, V. J., Flecknell, P. A., Gaffan, D., Garrod, K., Lemon, R. N., et al. (2010). Refinement of the use of food and fluid control as motivational tools for macaques used in behavioural neuroscience research: report of a Working Group of the NC3Rs. J. Neurosci. Met. 193, 167-188. doi: 10.1016/j.jneumeth.2010.09.003

Raleigh, M. J., McGuire, M. T., Brammer, G. L., Pollack, D. B., and Yuwiler, A. (1991). Serotonergic mechanisms promote dominance acquisition in adult male vervet monkeys. Brain Res. 559, 181-190. doi: 10.1016/0006-8993(91)90001-C

Redmond, D. E., Maas, J. W., Kling, A., Graham, C. W., and Dekirmenjian, H. (1971). Social behavior of monkeys selectively depleted of monoamines. Science 174, 428-431. doi: 10.1126/science.174.4007.428

Remy, P., Doder, M., Lees, A., Turjanski, N., and Brooks, D. (2005). Depression in Parkinson's disease: loss of dopamine and noradrenaline innervation in the limbic system. Brain 128, 1314-1322. doi: 10.1093/brain/awh445 
Riddick, N. V., Czoty, P. W., Gage, H. D., Kaplan, J. R., Nader, S. H., Icenhower, M., et al. (2009). Behavioral and neurobiological characteristics influencing social hierarchy formation in female cynomolgus monkeys. Neuroscience 158, 1257-1265. doi: 10.1016/j.neuroscience.2008.11.016

Roeltgen, D. P., and Schneider, J. S. (1991). Chronic low-dose MPTP in nonhuman primates: a possible model for attention deficit disorder. J. Child. Neurol. 6, 82-89.

Rushworth, M. F., Behrens, T. E., Rudebeck, P. H., and Walton, M. E. (2007). Contrasting roles for cingulate and orbitofrontal cortex in decisions and social behaviour. Trends Cogn. Sci. 11, 168-176. doi: 10.1016/j.tics.2007.01.004

Schneider, J. S. (1990). Chronic exposure to low doses of MPTP. II. Neurochemical and pathological consequences in cognitively-impaired, motor asymptomatic monkeys. Brain Res. 534, 25-36. doi: 10.1016/0006-8993(90)90108-N

Schneider, J. S. (2006). "Modeling cognitive deficits associated with Parkinsonism in the chronic-low-dose MPTP-treated monkey," in Animal Models of Cognitive Impairment, eds E. D. Levin and J. J. Buccafusco (Boca Raton, FL: CRC Press), Chapter 9, Frontiers in Neuroscience.

Schneider, J. S., and Kovelowski, C. J. II. (1990). Chronic exposure to low doses of MPTP. I. Cognitive deficits in motor asymptomatic monkeys. Brain Res. 519, 122-128. doi: 10.1016/0006-8993(90)90069-N

Schneider, J. S., and Pope-Coleman, A. (1995). Cognitive deficits precede motor deficits in a slowly progressing model of parkinsonism in the monkey. Neurodegeneration 4, 245-255. doi: 10.1016/1055-8330(95)90014-4

Schneider, J. S., and Roeltgen, D. P. (1993). Delayed matching-to-sample, object retrieval, and discrimination reversal deficits in chronic low dose MPTP-treated monkeys. Brain Res. 615, 351-354. doi: 10.1016/0006-8993(93)90049-S

Schneider, J. S., Tinker, J. P., and Decamp, E. (2010). Clonidine improves attentional and memory components of delayed response performance in a model of early Parkinsonism. Behav. Brain Res. 211, 236-239. doi: 10.1016/j.bbr.2010. 03.040

Schrag, A., Jahanshahi, M., and Quinn, N. (2000). How does Parkinson's disease affect quality of life? A comparison with quality of life in the general population. Mov. Dis. 15, 1112-1118. doi: 10.1002/1531-8257(200011)15:6<1112::AIDMDS1008>3.0.CO;2-A

Siever, L. J. (2008). Neurobiology of aggression and violence. Am. J. Psychiatr. 165, 429-442. doi: 10.1176/appi.ajp.2008.07111774

Skuse, D. H., and Gallagher, L. (2009). Dopaminergic-neuropeptide interactions in the social brain. Trends Cogn. Sci. 13, 27-35. doi: 10.1016/j.tics.2008.09.007

Sueur, C., Jacobs, A., King, A. J., Petit, O., and Amblard, F. (2011). How can social network analysis improve the study of primate behaviour? Am. J. Primatol. 73, 703-719. doi: 10.1002/ajp.20915

Suzuki, A., Hoshino, T., Shigemasu, K., and Kawamura, M. (2006). Disgust-specific impairment of facial expression recognition in Parkinson's disease. Brain 129, 707-717. doi: 10.1093/brain/awl011

Taylor, J. R., Roth, R. H., Sladek, J. R., and Redmond, D. E. (1990). Cognitive and motor deficits in the performance of an object retrieval task with a barrierdetour in monkeys (Cercopithecus aethiops sabaeus) treated with MPTP: longterm performance and effect of transparency of the barrier. Behav. Neurosci. 104, 564-576. doi: 10.1037/0735-7044.104.4.564

Thobois, S., Ardouin, C., Lhommée, E., Klinger, H., Lagrange, C., Xie, J., et al. (2010). Non-motor dopamine withdrawal syndrome after surgery for Parkinson's disease: predictors and underlying mesolimbic denervation. Brain 133, 1111-1127. doi: 10.1093/brain/awq032
Tzschentke, T. M. (2001). Pharmacology and behavioral pharmacology of the mesocortical dopamine system. Prog. Neurobiol. 63, 241-320. doi: 10.1016/S0301-0082(00)00033-2

Ulla, M., Thobois, S., Llorca, P. M., Derost, P., Lemaire, J. J., Chereau-Boudet, I., et al. (2012). Contact dependent reproducible hypomania induced by deep brain stimulation in Parkinson's disease: clinical, anatomical and functional imaging study. Behav. Neurol. 82, 607-614. doi: 10.1136/jnnp.2009.199323

Van Hooff, J. A. R. A. M. (1967). "The facial displays of the catarrhine monkeys and apes," in Primate Ethology, ed D. Morris (London: Weidenfeld and Nicolson), $7-68$.

Van Noordwijk, M. A., and Van Schaik, C. P. (1987). Competition among female long-tailed macaques, Macaca fascicularis. Anim. Behav. 35, 577-589. doi: 10.1016/S0003-3472(87)80284-1

Van Noordwijk, M. A., and Van Schaik, C. P. (1999). The effects of dominance rank and group size on female lifetime reproductive success in wild longtailed macaques, Macaca fascicularis. Primates 40, 105-130. doi: 10.1007/BF025 57705

Vezoli, J., Fifel, K., Leviel, V., Dehay, C., Kennedy, H., Cooper, H. M., et al. (2011). Early presymptomatic and long-term changes of rest activity cycles and cognitive behavior in a MPTP-monkey model of Parkinson's disease. PLoS ONE 6:e23952. doi: 10.1371/journal.pone.0023952

Videnovic, A., Lazar, A. S., Barker, R. A., and Overeem, S. (2014). 'The clocks that time us'-circadian rhythms in neurodegenerative disorders. Nat. Rev. Neurol. 10, 683-693. doi: 10.1038/nrneurol.2014.206

Weintraub, D., Siderowf, A. D., Potenza, M. N., Goveas, J., Morales, K. H., Duda, J. E., et al. (2006). Association of dopamine agonist use with impulse control disorders in Parkinson disease. Arch. Neurol. 63, 969-973. doi: 10.1001/archneur.63.7.969

Whitehead, H. (2009). SOCPROG programs: analyzing animal social structures. Behav. Ecol. Sociobiol. 63, 765-778. doi: 10.1007/s00265-008-0697-y

Yoshimura, N., Kawamura, M., Masaoka, Y., and Homma, I. (2005). The amygdala of patients with Parkinson's disease is silent in response to fearful facial expressions. Neuroscience 131, 523-534. doi: 10.1016/j.neuroscience.2004. 09.054

Conflict of Interest Statement: The authors declare that the research was conducted in the absence of any commercial or financial relationships that could be construed as a potential conflict of interest.

Received: 17 November 2014; accepted: 05 February 2015; published online: 26 February 2015.

Citation: Durand E, Petit O, Tremblay L, Zimmer C, Sgambato-Faure V, Chassain C, Laurent $M$, Pereira B, Silberberg $C$ and Durif F (2015) Social behavioral changes in MPTP-treated monkey model of Parkinson's disease. Front. Behav. Neurosci. 9:42. doi: 10.3389/fnbeh.2015.00042

This article was submitted to the journal Frontiers in Behavioral Neuroscience. Copyright (c) 2015 Durand, Petit, Tremblay, Zimmer, Sgambato-Faure, Chassain, Laurent, Pereira, Silberberg and Durif. This is an open-access article distributed under the terms of the Creative Commons Attribution License (CC BY). The use, distribution or reproduction in other forums is permitted, provided the original author(s) or licensor are credited and that the original publication in this journal is cited, in accordance with accepted academic practice. No use, distribution or reproduction is permitted which does not comply with these terms. 